\title{
Conf-9407166--2
}

UCRL- JC- 119193

PREPRINT

\section{The Uses of Electron Beam Ion Traps in the Study of Highly Charged Ions}

D. Knapp

This paper was prepared for Submittal to Physics with Multiply Charged Ions Proceedings

July 18-30, 1994, Cargese, Corsica

November 2, 1994

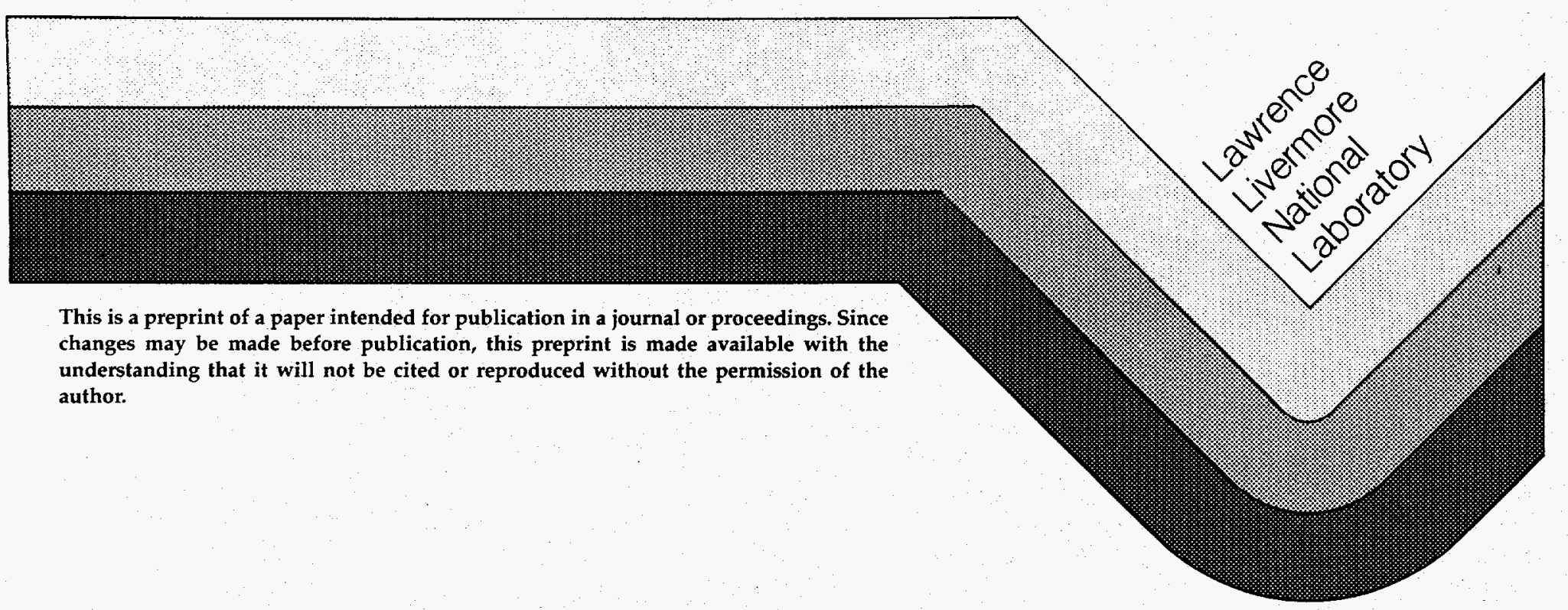




\section{DISCLAIMER}

This document was prepared as an account of work sponsored by an agency of the United States Government. Neither the United States Government nor the University of California nor any of their employees, makes any warranty, express or implied, or assumes any legal liability or responsibility for the accuracy, completeness, or usefulness of any information, apparatus, product, or process disdosed, or represents that its use would not infringe privately owned rights. Reference herein to any specific commercial products, process, or service by trade name, trademark, manufacturer, or otherwise, does not necessarily constitute or imply its endorsement, recommendation, or favoring by the United States Government or the University of California. The views and opinions of authors expressed herein do not necessarily state or reflect those of the United States Government or the University of Califomia, and shall not be used for advertising or product endorsement purposes. 


\section{DISCLAIMER}

Portions of this document may be illegible in electronic image products. Images are produced from the best available original document. 


\title{
THE USES OF ELECTRON BEAM ION TRAPS IN
}

\section{THE STUDY OF HIGHLY CHARGED IONS}

\author{
David A. Knapp \\ Physics Department \\ Lawrence Livermore National Laboratory \\ Livermore, CA 94550 USA
}

\section{INTRODUCTION}

The Electron Beam Ion Trap (EBIT) is a relatively new tool for the study of highly charged ions. Its development has led to a variety of new experimental opportunities; measurements have been performed with EBITs using techniques impossible with conventional ion sources or storage rings. In this paper, I will highlight the various experimental techniques we have developed and the results we have obtained using the EBIT and higherenergy Super-EBIT built at the Lawrence Livermore National Laboratory.

The EBIT employs a high-current-density electron beam to trap, ionize, and excite a population of ions. ${ }^{2}$ The ions can be studied in situ or extracted from the trap for external experiments. The trapped ions form an ionization-state equilibrium determined by the relative ionization and recombination rates. Ions of several different elements may simultaneously be present in the trap. The ions are nearly at rest, and, for most systems, all in their ground-state configurations. The electron-ion interaction energy has a narrow distribution and can be varied over a wide range. We have used the EBIT devices for the measurement of electron-ion interactions, ion structure, ion-surface interactions, and the behavior of lowdensity plasmas.

\section{THE EBIT DEVICES}

The EBIT was originally designed as a variation of an Electron Beam Ion Source (EBIS) optimized for spectroscopy applications. The basic principle of EBIT operation is illustrated in Figure 1. An electron beam, compressed and confined by a magnetic field, passes through a series of three drift tubes. The ions are trapped radially by the space charge of the electron beam, and axially by voltages applied to the end drift tubes. The electron beam also serves to ionize and excite the ions via inelastic collisions.

The EBITs differ in several ways from EBISes. First, and most important, they are much shorter. EBISes generally consist of an electron beam traveling through several drift tubes in a solenoidal magnetic field; the typical trap length of an EBIS is of the order of $1 \mathrm{~m}$. 


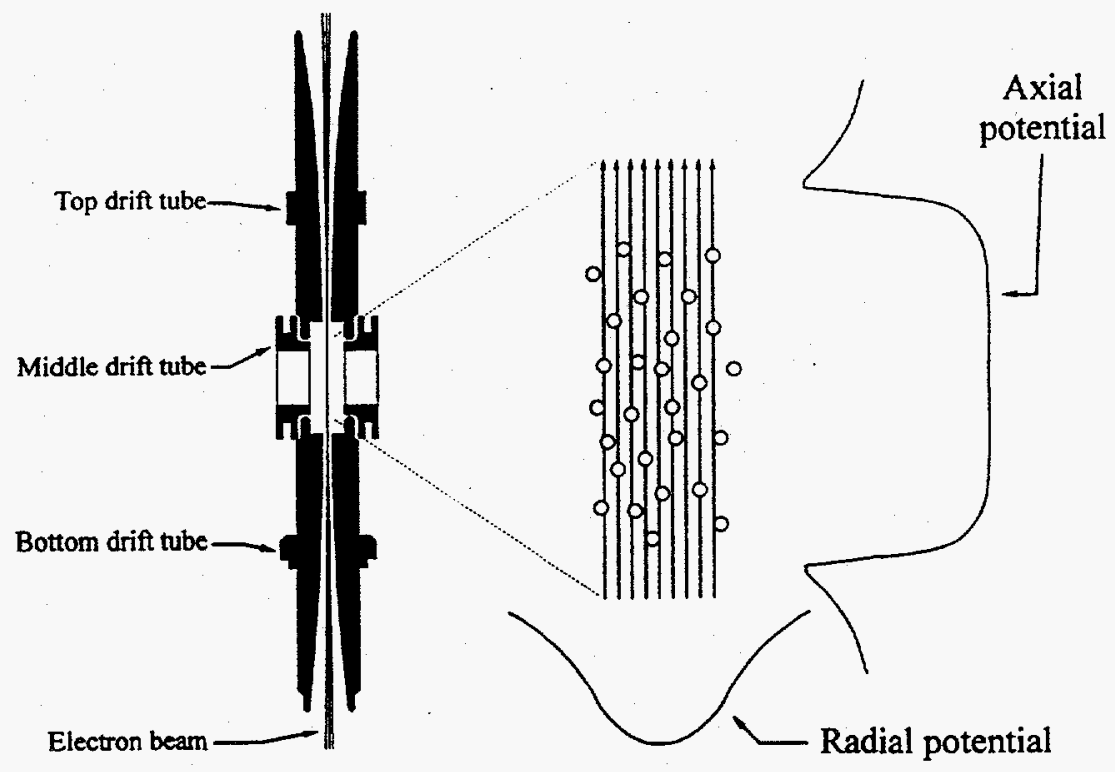

Figure 1. Basic operation of the EBIT trap. A high-current-density electron beam, compressed by a magnetic field, passes through three drift tubes. Ions are radially confined by the space charge of the beam; they are confined axially by voltages applied to the drift tubes.

In an EBIT, the trap is confined to the length of a single drift tube (about $2 \mathrm{~cm}$ ), and the magnetic field is generated by a Helmholtz coil pair. The shorter trap results in much reduced amplitudes for plasma instabilities, which heat the ions and drive them out of the electron beam. The Helmholtz coils allow viewing the ions from the side of the trap, greatly increasing the available solid angle. Second, the EBIT has a much higher magnetic field and electron current density. This allows faster ionization of the atoms in the trap, and higher interaction rates with the beam electrons. Finally, most EBISes require a continuous source of gaseous atoms from which to form ions; the EBIT device can form or capture and ionize low-charge ions from a variety of sources.

\section{EBIT Design}

The general design scheme for the EBIT electron beam path is shown in Figure 2. The electron beam originates in an electron gun at ground potential with zero magnetic field at the cathode. It is accelerated into the drift tubes and greatly compressed by an axial magnetic field. The drift tubes float at voltages up to about $30 \mathrm{kV}$. The electron beam path terminates in a radial collector floating at a voltage of about $1.5 \mathrm{kV}$. The magnetic field vanishes in the collector, allowing the beam to expand and the electrons to be collected on the walls. Suppresser electrodes between the drift tubes and collector prevent secondary electrons emitted in the collector from entering the drift tube region.

After the successful construction and operation of two EBITs, the original device was upgraded to provide a higher interaction energy. The modified EBIT, called SuperEBIT, achieves a higher electron beam energy by having both the electron gun and the collector float at a negative voltage. ${ }^{2}$ Layout drawings of EBIT and SuperEBIT are shown in Figure 3. The new collector in Super-EBIT is located in a separate vacuum vessel because the original EBIT vessel was not large enough for the high-voltage gap required. The collector's position necessitated an increase in the electron path length of about $1 \mathrm{~m}$. Addi- 


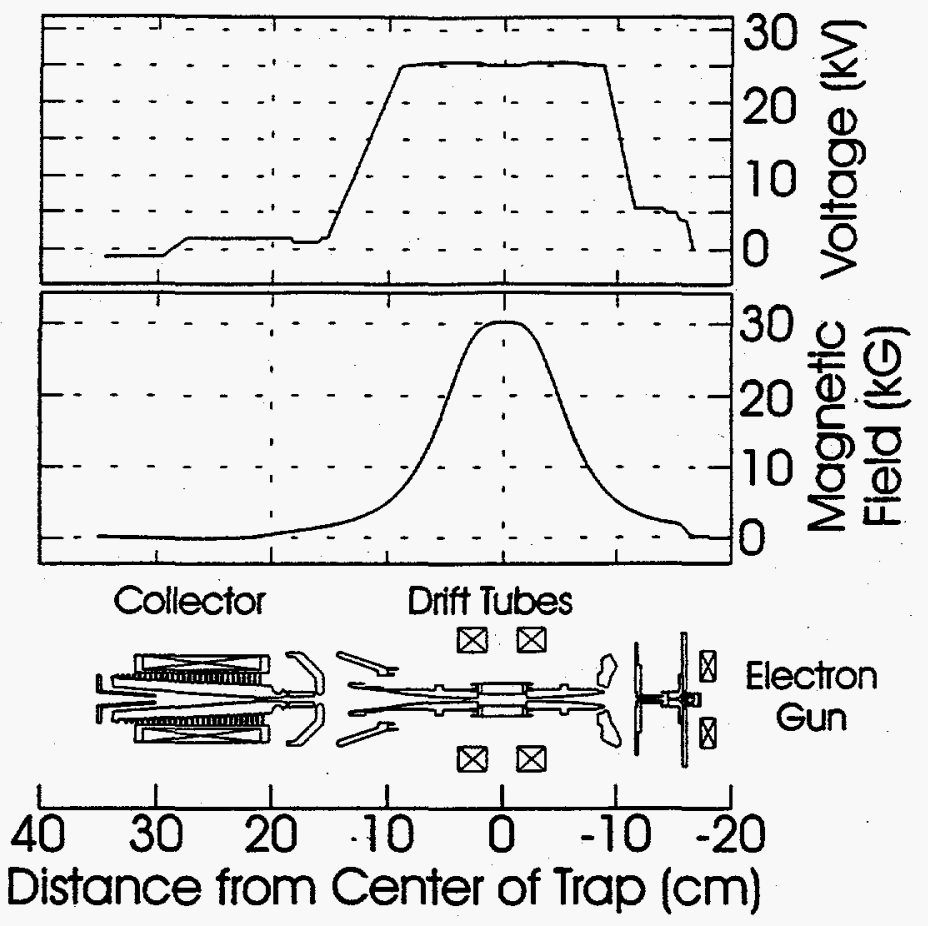

Figure 2. Design scheme for EBIT, showing the electron beam energy and magnetic field values along the electron beam trajectory. The electron beam travels from the electron gun, which is at ground potential, through the drift tubes, which are at voltages up to $30 \mathrm{kV}$, and into the collector, which is at about $1.5 \mathrm{kV}$.

tional modification of the drift tubes to eliminate breakdowns induced by trapped Penning electrons has extended their operating voltage range to approximately $40 \mathrm{kV}$. Super-EBIT has successfully run at electron-ion interaction energies as high as $205 \mathrm{keV}$. With the addition of an $\mathrm{SF}_{6}$ atmosphere for high-voltage insulation on the air side of the electron-gun high-voltage feedthrough, we anticipate future operation at energies up to $250 \mathrm{keV}$.

Typical EBIT and Super-EBIT operating parameters are shown in Table 1. Because of its long electron beam path, Super-EBIT does not function well at electron energies be-

Table 1. Typical EBIT and Super-EBIT operating parameters. The electron beam radii were obtained by imaging the electron beam through a slit with a beam current of $150 \mathrm{~mA}$. The trapping time in Super-EBIT is presently limited by intermittent high-voltage breakdowns.

\begin{tabular}{|l|c|c|c|}
\hline \multicolumn{1}{|c|}{ Parameter } & Symbol & $\begin{array}{c}\text { Value for } \\
\text { EBIT }\end{array}$ & $\begin{array}{c}\text { Value for } \\
\text { Super-EBIT }\end{array}$ \\
\hline Electron-Ion Interaction Energy & $E_{0}$ & $.5-25 \mathrm{keV}$ & $10-200 \mathrm{keV}$ \\
Electron Beam Current & $I_{e}$ & $0-160 \mathrm{~mA}$ & $0-200 \mathrm{~mA}$ \\
Electron Beam Radius in Trap & $r_{\mathrm{e}}$ & $40 \mu \mathrm{m}$ & $35 \mu \mathrm{m}$ \\
Effective Electron Current Density & $\left\langle j_{e}\right\rangle$ & $0-3200 \mathrm{~A} / \mathrm{cm}^{2}$ & $0-4000 \mathrm{~A} / \mathrm{cm}^{2}$ \\
Trap Length & & $2 \mathrm{~cm}$ & $2 \mathrm{~cm}$ \\
Magnetic Field in Trap & $B_{\text {TRAP }}$ & $3 \mathrm{~T}$ & $3 \mathrm{~T}$ \\
Axial Trap Depth & $V_{\text {TRAP }}$ & $10-400 \mathrm{~V}$ & $10-400 \mathrm{~V}$ \\
Highest Uranium Ionization State & $q_{\text {MAX }}$ & $U^{82+}($ neonlike) & $U^{92+}($ bare $)$ \\
Total Number of Trapped Ions & $N_{\text {TOT }}$ & $10^{5}-10^{6}$ & $10^{5}-10^{6}$ \\
Ion Density & $n_{\text {TOT }}$ & $10^{9}-10^{10} \mathrm{~cm}^{-3}$ & $10^{9}-10^{10} \mathrm{~cm}^{-3}$ \\
Trapping Time & $\tau_{q}$ & $<5 \mathrm{~h}$ & $<2 \mathrm{~h}$ \\
\hline
\end{tabular}


low $10 \mathrm{keV}$; but can operate at very high current for high electron beam energies. The relatively short trapping times indicated for Super-EBIT result from intermittent high-voltage breakdowns, not from any inherent limitation of the trap.

\section{Introduction of Ions into EBIT}

Ions are introduced into the EBIT traps in one of four ways. Atoms evaporated from the electron gun cathode naturally find their way into the beam, become ionized, and are trapped. These atoms are primarily barium, with smaller amounts of tungsten and osmium. The gas injection system is used for normally gaseous elements, and can have an oven attached for $\mathrm{Ti}, \mathrm{Na}$, and similar metals with high vapor pressures. Most ions from

(b)

(a)
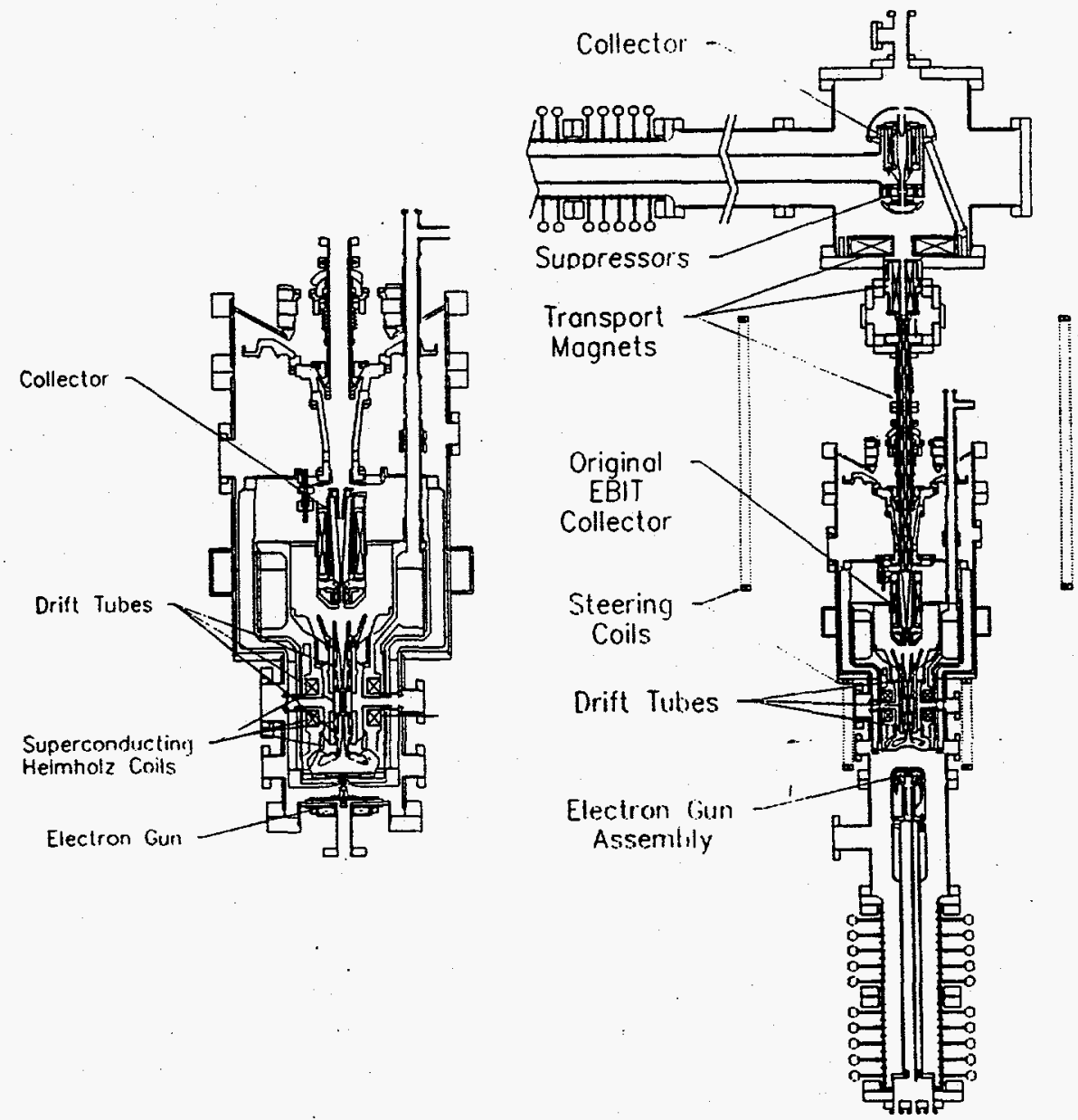

Figure 3. Layout designs for (a) EBIT and (b) Super-EBIT. The drift-tube geometry for the two devices is identical. In Super-EBIT, both the electron gun and collector float at negative high voltage, allowing the electron beam energy in the trap to be much higher. 
metallic solids are injected from a Metal Vapor Vacuum Arc (MEVVA) source located above the trap. These ions are injected along the electron beam, and captured by raising the top drift tube voltage while the ion pulse is in the trap. We have also successfully used the MEVVA source to inject ions from sintered materials comprised of metal oxides in a metallic copper matrix.

Steven Elliott and Ross Marrs have recently developed a new technique for introducing ions from very small samples into the trap. ${ }^{3}$ The material is placed on the end of a small wire, which is introduced radially into the periphery of the electron beam. Atoms sputtered from the sample and ionized by electron collisions are captured in the trap.

\section{Ionization Balance in EBIT}

The primary interactions that affect the charge balance in EBIT are electron-ion interactions and charge exchange with neutral gas atoms. Beam electrons interact to change the ionization state of the ions by ionization, radiative recombination, and dielectronic recombination. Dielectronic recombination is a resonant process, however, and at most operating energies it is not a significant factor in the determination of the equilibrium charge balance. Therefore, for electron-beam energies not on dielectronic recombination resonances, assuming that the interactions are dominated by single-charge-changing processes, the time evolution for the population of charge state $q$ of a given element $Z$ is given by:

$$
\frac{d N_{q}}{d t}=\frac{\left\langle j_{e}\right\rangle}{\mathrm{e}}\left[\left(\sigma_{q-1}^{1} N_{q-1}+\sigma_{q+1}^{\mathrm{RR}} N_{q+1}\right)-\left(\sigma_{q}^{\mathrm{I}}+\sigma_{q}^{\mathrm{RR}}\right) N_{q}\right]+n_{0} v_{0}\left[\sigma_{q+1}^{\mathrm{CX}} N_{q+1}+\sigma_{q}^{\mathrm{CX}} N_{q}\right]
$$

where $N_{q}$ is the number of ions in charge state $q,\left\langle j_{0}\right\rangle$ is the effective current density of the electron beam, $\sigma_{q}^{\mathrm{I}}, \sigma_{q}^{\mathrm{RR}}$, and $\sigma_{q}^{\mathrm{X}}$ are the cross sections for ionization, radiative recombina-
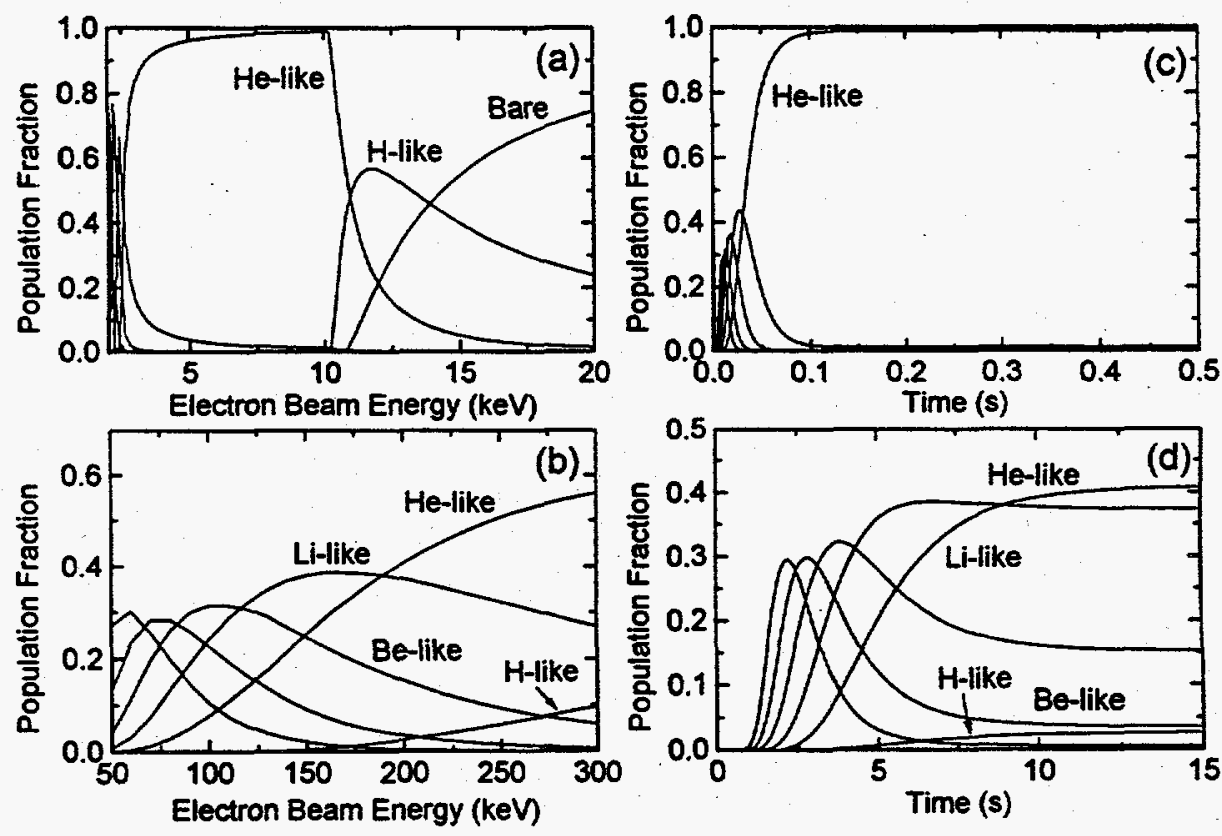

Figure 4. (a) and (b): Predicted equilibrium ionization balances in an EBIT for (a) nickel and (b) uranium ions over energy ranges of interest. The ionization balances were calculated assuming that the charge-exchange recombination is negligible. (c) and (d): Predicted time evolution of ionization balances. (c) Nickel ions at $E_{\mathrm{e}}=10 \mathrm{keV}$. (d) Uranium ions at $E_{\mathrm{e}}=200 \mathrm{keV}$. 
tion, and charge-exchange recombination, respectively, for charge state $q$, and the neutral gas density and velocity are given by $n_{0}$ and $v_{0}$.

We obtain a predicted ionization balance by setting $d N_{d} / d t=0$ for all $q$ and solving the resulting simultaneous equations. We use the Lotz formula to approximate the ionization cross section, and the theory of $\mathrm{Kim}$ and Pratt ${ }^{3}$ for the radiative recombination. Predicted ionization balances for nickel and uranium ions are shown in Figure $4 a$ and $b$. Ions with closed $n$ shells are the easiest to produce in a relatively pure ionization state because of the large change in ionization energy that occurs at a closed shell. Thus, heliumlike $\mathrm{Ni}^{26+}$ dominates the ionization balance at energies below the $n=1$ ionization energy.

Estimates of the time evolution of the ionization balances are also shown in Figure $4 \mathrm{c}$ and $\mathrm{d}$. Depending on the ion species, the equilibration time varies over a wide range, from less than $100 \mathrm{~ms}$ for light ions to more than $20 \mathrm{sec}$ for hydrogenic and bare uranium.

\section{Ion Trapping Times}

The observed trapping times for ions in EBIT are much longer than was originally predicted. Ion-electron collisions heat the ions; in order to achieve long trapping times, this energy must be removed via some cooling mechanism. We have determined that the ions in EBITs undergo a process of "evaporative cooling" in which low- $Z$ ions preferentially escape the trap, cooling high-charge, high- $Z$ ions. ${ }^{6}$ This process is illustrated in Figure 5 . In a trap of depth $V_{\text {TRAP }}$, ions of charge q need an energy of to escape. In an EBIT, the effective radial trap depth is very large because of the high magnetic field, so the ions escape axially. For all ions, , so for ions at the same temperature (and thus the same energy per ion), high$\mathrm{q}$, high- $Z$ ions are trapped much more deeply than are low- $Z$ ions. In an EBIT, ion-ion collisions tend to equalize the temperatures of all ions, so the loss of low-q ions cools the high$q$ ions. The final required ingredient for the long-term cooling of the high- $q$ ions is a constant influx of low- $q$ ions. In EBIT, a gas injection system continuously introduces a small number of ballistic low- $Z$ atoms into the trap, where they are ionized by the beam and cool

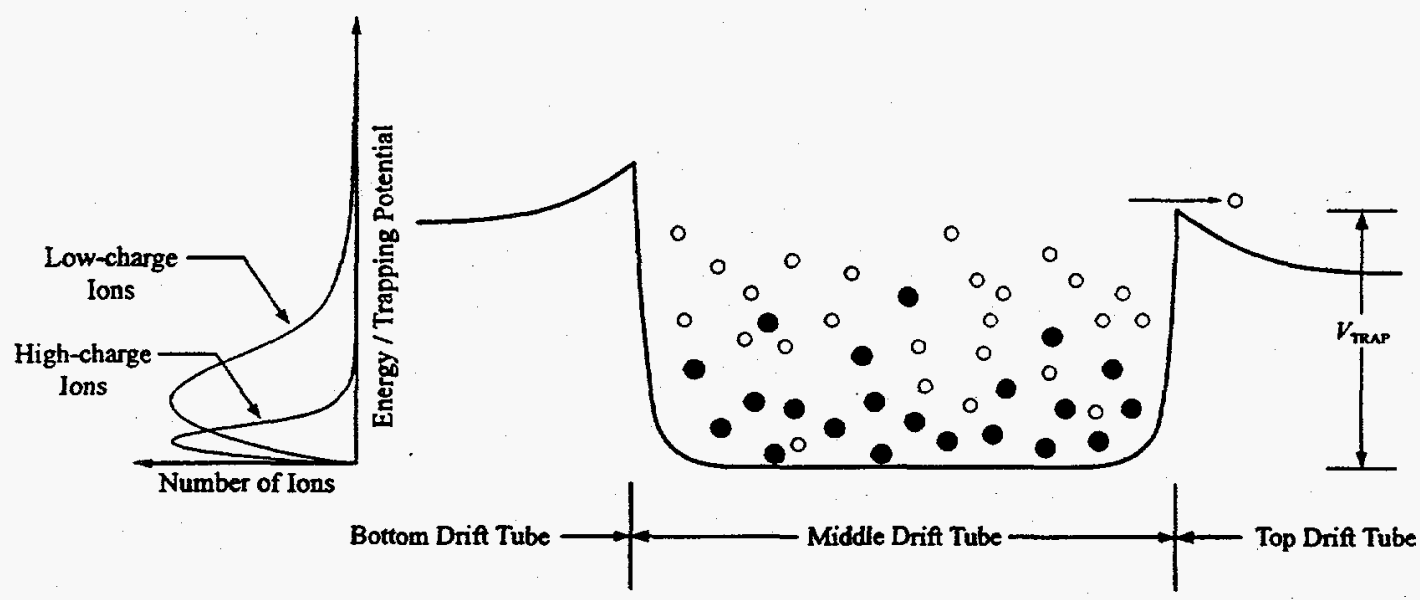

- High-charge ions (charge $Q$ ) trapped by $\mathrm{V}_{\mathrm{TRAP}} \times Q$

- Low-charge ions (charge $q$ ) trapped by $V_{\text {Trap }} \times q$

Figure 5. Evaporative cooling in an electron beam ion trap. High-charge jons with charge $Q$ are trapped by a potential of $Q V_{\mathrm{TRAP}}$, while ions of charge state $q<\ll Q$ are trapped by a potential of $q V_{\text {TrAp. }}$ Ion-ion collisions bring the energy distributions of the two ion species into equilibrium at the same temperature, so the low-charge ions preferentially escape the trap, cooling the high-charge ions. 
the higher- $Z$ ions in the trap.

Although the ballistic gas atoms cool the ions in the trap, the number density of background gas atoms must be carefully controlled to prevent charge-exchange recombination from negatively affecting the ionization balance in the trap. This problem is especially crucial in the operation of Super-EBIT, because the charge-exchange recombination cross sections are quite large for the highest charge ions. As a result, the Super-EBIT trap region is pumped by an array of $4 \mathrm{~K}$ charcoal surfaces, and, with the exception of the gas injection port, all ports into the trap are covered by radiation windows. The background gas contamination from the gas injection port is minimized by use of a narrow tube that only allows ballistic gas atoms from the source into the trap region.

\section{EBIT Operation}

The processing of ions in EBIT requires a complex timing arrangement to take the device through injection, ionization, observation, and extraction. The drift tube voltage, electron beam current, trap depth, and many other parameters must be precisely synchronized. We have developed a system that allows easy programming of this precise timing for cycles lasting from less than $1 \mathrm{~s}$ to more than $1000 \mathrm{~s}$.

A "typical" EBIT experiment follows a timing sequence as follows: ions are generated in the MEVVA source and injected into the trap by along the electron beam direction. The top drift tube voltage is lowered to allow the ions to enter the trap. The drift tube voltage is adjusted to minimize the velocity of the ions in the trap. As the ions reflect from the potential of the bottom drift tube, the voltage of the top drift tube is quickly raised, trapping the ions. The ions are then allowed to ionize for a time until the ionization balance comes to equilibrium. The electron-ion interaction energy is then changed to the "probe energy," which may either be constant or a function of time. During this phase, the photons emitted by the ions are observed by a variety of detectors. Finally, the drift tube voltage is set to the desired value for extraction, and the ions are ejected from the trap into the ion transport system.'

\section{SPECTROSCOPY EXPERIMENTS}

The largest class of experiments performed using the EBIT has been the measurement of $x$-ray spectra of highly charged ions. From these studies, the structure of the ions can be deduced. EBITs have several advantages over other devices that produce highlycharged ions for spectroscopy:

1. The ions in an EBIT are essentially at rest, with a temperature typically less than $100 \mathrm{q} \mathrm{eV}$. No Doppler shift corrections need to be applied.

2. The EBIT trap forms a line source; it is about $2 \mathrm{~cm}$ long and $30 \mu \mathrm{m}$ in radius. It is thus an excellent source for diverse spectrometer geometries.

3. The EBIT source has no equilibrium population of metastable states, with the exception of very rare states with lifetimes greater than $0.1 \mathrm{~s}$.

4. The EBIT source is a low-density, weak-field plasma; for most systems, collisional processes, Stark effects, and magnetic field effects can be neglected, and the absorption of emission lines is negligible.

These properties make EBIT a nearly ideal source for high-resolution spectroscopy.

The LLNL EBITs have been used for a wide variety of spectroscopy experiments. Most of these have employed high-resolution crystal spectrometers, in which $x$ rays meeting the Bragg condition are reflected from a crystal into a position-sensitive detector. ${ }^{8}$ I will summarize a few of the most interesting and important. 


\section{Observation of Magnetic Octupole Radiation}

The first observation of a magnetic octupole (M3) transition was achieved by Peter Beiersdorfer and his coworkers in nickel-like $\mathrm{U}^{64+}$ and $\mathrm{Th}^{65+}$ ions produced in the LLNL EBIT. ${ }^{9}$ A partial level diagram of the $\mathrm{U}^{64+}$ ion is shown in Figure 6a. The M3 transition rate is calculated to be $1.3 \times 10^{5} \mathrm{~s}^{-1}$, much slower than that of the neighboring E2 transition, with a transition rate of $3.3 \times 10^{10} \mathrm{~s}^{-1}$. The states in question are primarily populated by cascades rather than direct excitation. Observation of the $\mathrm{M} 3$ transition requires a low-density plasma, as its emission is very sensitive to collisional effects; because the transition rate is so low, electrons could be excited out of the state via collisions before the transition occurred. Calculations indicated that an electron density less than $10^{14} \mathrm{~cm}^{-3}$ was required to observe the transition.

$\mathrm{X}$ rays emitted from EBIT were analyzed by a $\mathrm{Ge}(111)$ flat-crystal spectrometer and detected by a position-sensitive proportional counter. The wavelengths were calibrated using the known $\mathrm{Ly}_{\alpha}$ lines from hydrogenic sulfur. A high-resolution spectrum of the $\mathrm{x}$ rays from $U^{64+}$ is shown in Figure $6 \mathrm{~b}$. The transition energies of the lines were measured to an accuracy of $0.2 \mathrm{eV}$, or a relative precision of $80 \mathrm{ppm}$. The M3 transition is seen clearly resolved from the E2 transition. The ratio of intensity of the $M 3$ and E2 lines was measured as a function of electron beam energy. Although calculations including cascades from $n \leq 5$ levels predicted that the ratio would decrease with increasing energy, the opposite was observed.

\section{X-Ray Laser Photopumping Schemes}

Present $\mathrm{x}$-ray lasers using neonlike ions, in which the $2 p^{5} 3 p$ state attains a population inversion relative to the $2 p^{5} 3 s$ state, rely on collisional excitation of the ions. ${ }^{10}$ The use of resonant photopumping as an alternative method for excitation of the ions offers the possi-

(a)

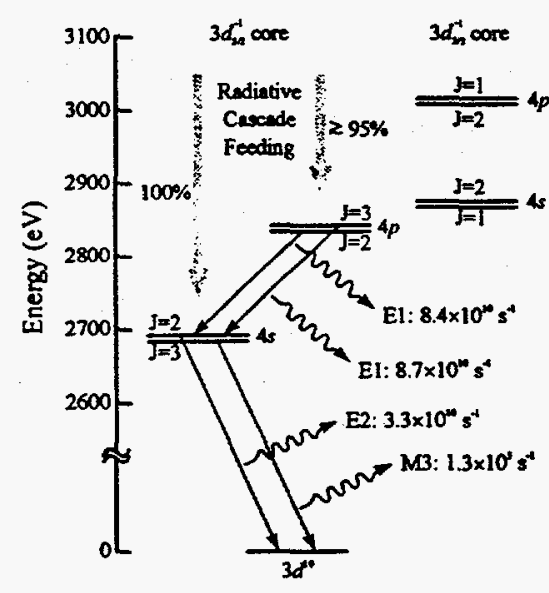

(b)

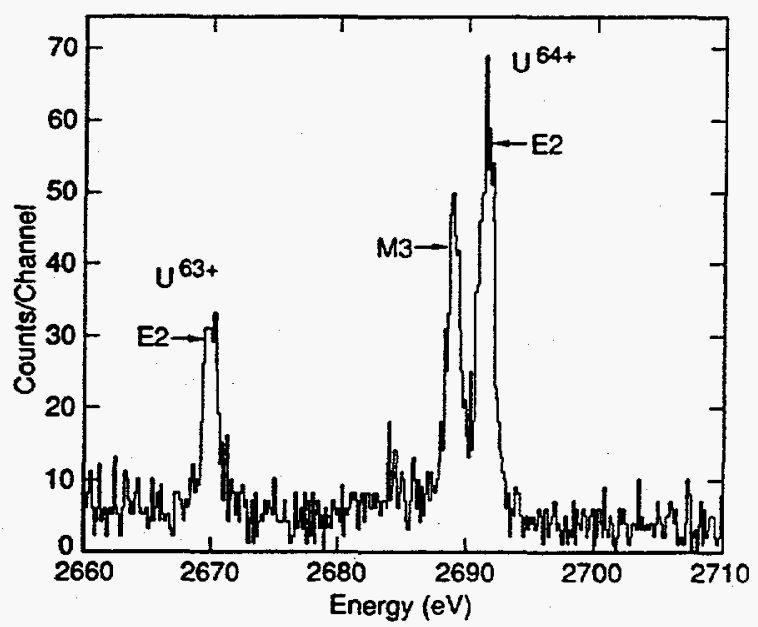

Figure 6. (a) Partial energy-level diagram for nickel-like $U^{64+}$, (b) High-resolution spectrum of the region containing the $\mathrm{M} 3$ line in $\mathrm{U}^{64+}$. The $\mathrm{M} 3$ transition is clearly resolved from the neighboring $\mathrm{E}_{2}$ transition. 
(a)
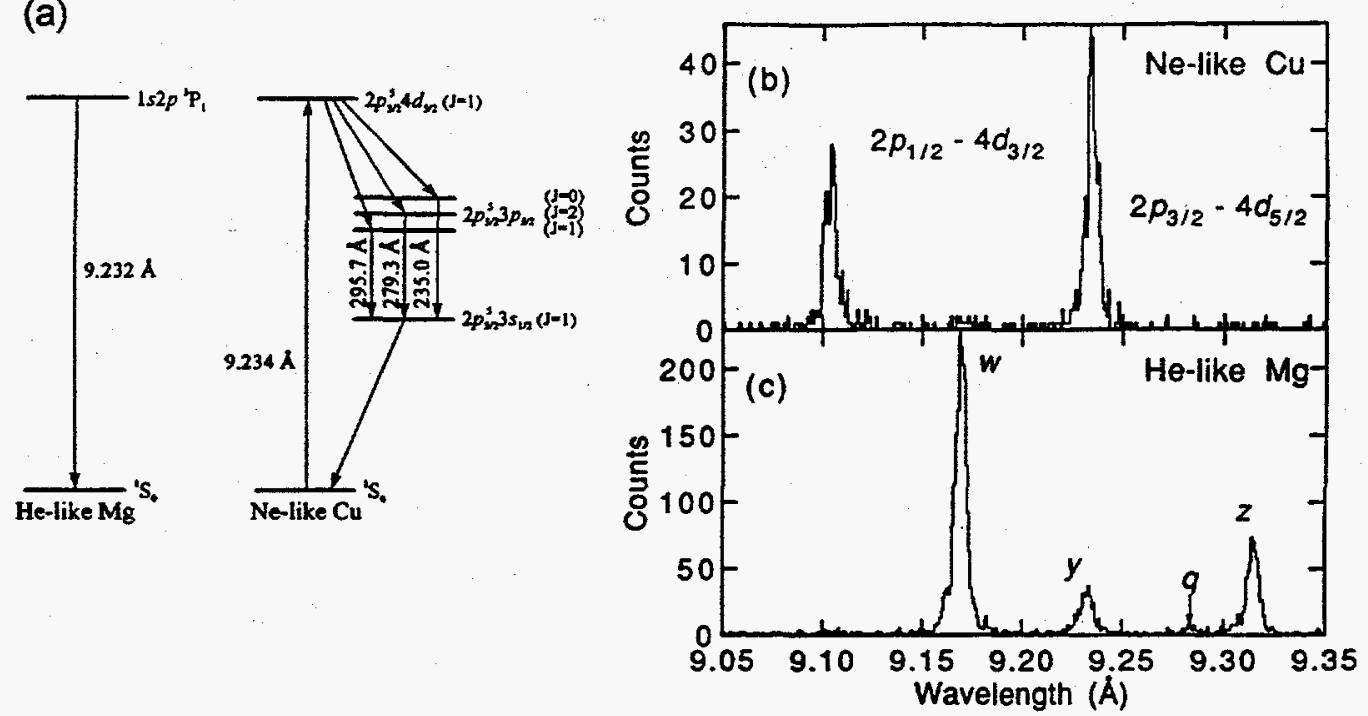

Figure 7. (a) Energy-level diagram for a photopumping scheme for $\mathrm{x}$-ray laser using neonlike $\mathrm{Cu}^{19+}$ and heliumlike $\mathrm{Mg}^{10+}$. (b) Measured spectrum of $\mathrm{Cu}^{194}$. (c) Measured spectrum of $\mathrm{Mg}^{10+}$.

bility of improved efficiency. ${ }^{11}$ Photopumped $\mathrm{x}$-ray lasing has not yet been experimentally observed, however, despite efforts to resonantly photopump neonlike $\mathrm{Rb}^{27+}$ ions with nickellike $\mathrm{Pt}^{30+}$ and $\mathrm{Au}^{31+12}$

EBIT is an ideal environment to study possible photopumping schemes in lower- $Z$ ions, for which the plasma electron temperatures need not be as high. One such scheme, using heliumlike $\mathrm{Mg}^{10+}$ to photopump neonlike $\mathrm{Cu}^{19+}$, was recently studied by Peter Beiersdorfer and collaborators. ${ }^{13}$ An energy-level diagram illustrating the photopumping scheme is shown in Figure 7a. The $\mathrm{Mg} y\left(1 s 2 p^{3} \mathrm{P}_{1} \rightarrow 1 s^{2} \mathrm{~S}_{0}\right)$ line resonantly pumps the $\mathrm{Cu}$ $2 p_{3 / 2} \rightarrow 4 d_{5 / 2}$ transition, and the resulting excited state decays primarily to the $2 p_{3 / 2} 3 p_{3 / 2}$ levels, forming the population inversion.

The transition energies were measured using an evacuated flat-crystal $x$-ray spectrometer attached to the EBIT trap, with a resolution of $\Delta \lambda / \lambda=1200$ for these photons. The resulting spectra for the $\mathrm{Cu}$ and $\mathrm{Mg}$ ions are displayed in Figure $\mathrm{Tb}$ and $\mathrm{c}$. The spectrometer dispersion was calibrated using the heliumlike $\mathrm{Mg}$ lines $w\left(1 s 2 p^{1} \mathrm{P}_{1} \rightarrow 1 s^{2} \mathrm{~S}_{0}\right)$ and $z\left(1 s 2 s^{3} \mathrm{~S}_{1} \rightarrow 1 s^{2} \mathrm{~S}_{0}\right)$. The measured separation of the $\mathrm{Mg} y$ line and the $\mathrm{Cu} 2 \mathrm{p}_{3 / 2} \rightarrow 4 d_{5 / 2}$ line is measured to be $0.27 \pm 0.10 \mathrm{eV}$. Since in a typical 500-eV laser plasma, the $\mathrm{Cu}$ line will be broadened by $0.2 \mathrm{eV}$ and the $\mathrm{Mg}$ line by $0.5 \mathrm{eV}$, these transitions make an excellent candidate for a photopumped laser.

\section{Radiative Lifetime Measurements}

EBIT has been used to fill in a significant gap in the measurement of radiative lifetimes. Prior techniques have been able to measure lifetimes for $\tau<1 \mu$ s (by beam-foil techniques) and $\tau>1 \mathrm{~ms}$ (by retrapping ions). The decay of the $1 s 2 s^{3} \mathrm{~S}_{1}$ excited state in heliumlike $\mathrm{Ne}^{8+}$, with a lifetime around $100 \mu \mathrm{s}$, is inaccessible to both techniques. A new method for measuring such lifetimes has been developed by Brad Wargelin and Peter Beiersdorfer using EBIT as a source. ${ }^{14}$ 
In the new technique, the lifetime is determined from measurement of the time decay of the x-ray emission from a population of stationary ions. The ions were prepared in EBIT with the electron beam energy set to $960 \mathrm{eV}$, above the direct-excitation threshold for the $n=2-1$ transitions, but below the excitation threshold for any $\mathrm{n} \geq 3$ states. The beam energy was quickly switched to $750 \mathrm{eV}$, below the direct-excitation threshold and away from any dielectronic recombination resonances. Photons were recorded by a $\mathrm{Si}(\mathrm{Li})$ detector and a high-resolution flat-crystal evacuated spectrometer. The data-acquisition system resolved the time of detection of the photons with a resolution of $1.6 \mu \mathrm{s}$. Typical spectra taken using the crystal spectrometer for electron beam energies above and below threshold are shown in Figure 8a. The four heliumlike $n=2-1$ lines, designated $w\left(1 s 2 p{ }^{1} \mathrm{P}_{1} \rightarrow 1 s^{2}{ }^{1} \mathrm{~S}_{0}\right), x$ $\left(1 s 2 p^{3} \mathrm{P}_{2} \rightarrow 1 s^{2} \mathrm{~S}_{0}\right), y\left(1 s 2 p^{3} \mathrm{P}_{1} \rightarrow 1 s^{21} \mathrm{~S}_{0}\right)$, and $z\left(1 s 2 s^{3} \mathrm{~S}_{1} \rightarrow 1 s^{2}{ }^{1} \mathrm{~S}_{0}\right)$, along with the lithiumlike line $q\left(1 s 2 s 2 p^{2} \mathrm{P}_{3 / 2} \rightarrow 1 s^{2} 2 s^{2} \mathrm{~S}_{1 / 2}\right)$, are visible above threshold. Below threshold, only decays originating in the $1 s 2 s^{3} \mathrm{~S}_{1}$ state can be seen; line $z$ results from radiative decay of this state, and lines $x$ and $y$ are excited by $2 s-2 p$ electron impact excitations. The ratio of intensities $I_{x}+I_{y} / I_{z}$ is determined by the effective electron current density $\left\langle j_{e}\right\rangle$.

Measurements were made at three electron densities, which were determined from the intensity ratios. The lifetime was determined at each density from:

$$
\tau_{z}=\tau_{\text {decas }}\left[1+(1+k) \frac{I_{x}+I_{y}}{I_{z}}\right]
$$

where the constant $k=\tau_{c x} / \tau_{c 0} \ll 1$ is the ratio of collisional transfer to the states resulting in lines $x$ and $y$ to collisional transfer to the ground state. The derived lifetimes for the three densities agreed well, giving a combined value of $\tau_{z}=90.49 \pm 1.48 \mu \mathrm{s}$. This value is compared to theoretical predictions and other measurements in heliumlike ions in Figure $8 \mathrm{~b}$; the agreement with theory is good.

(a)

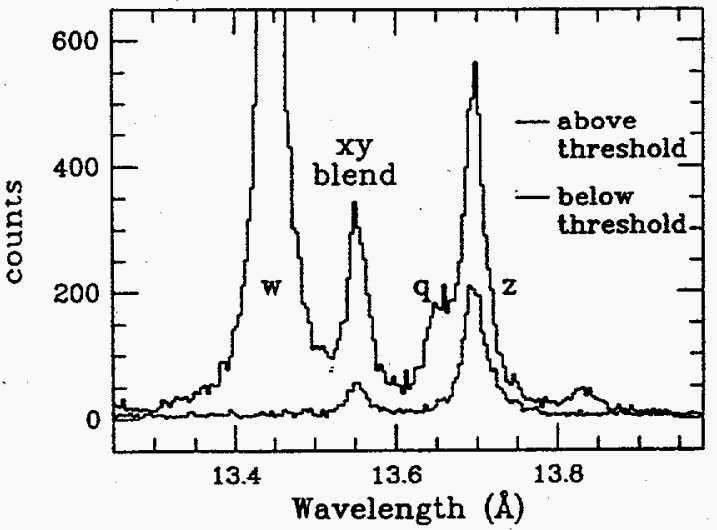

(b)

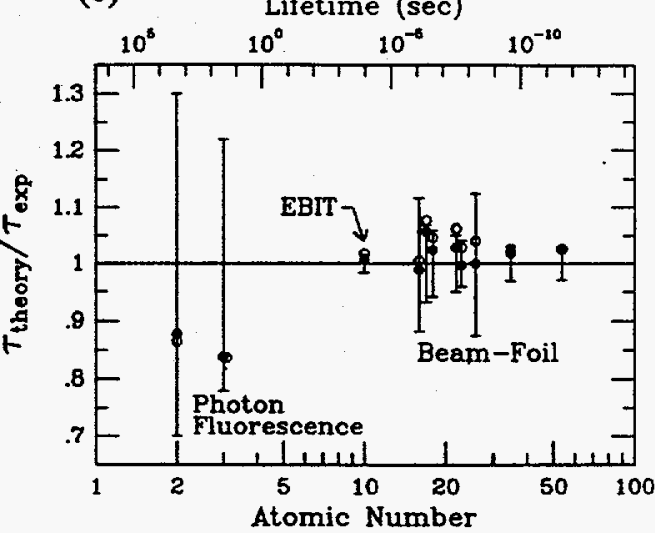

Figure 8. (a) Spectrum of heliumlike $\mathrm{Ne}^{8+}$ above and below the direct-excitation threshold for $\mathrm{n}=2-1$ transitions. (b) This lifetime result compared to theoretical predictions of Drake $e^{15}$ and Johnson and Lin. ${ }^{16}$ For the sources of the experimental points, see Ref. 14. 


\section{Measurements of $n=2-2$ Transitions in Highly-Charged Uranium Ions}

High-resolution spectroscopy of few-electron high- $Z$ ions is important for tests of quantum electrodynamics (QED) and relativistic calculations, as both contributions scale as $Z^{4}$. The width of the ionization distribution for high- $Z$ ions in Super-EBIT provides a unique opportunity to measure transition energies along isonuclear sequences. In uranium ions between the neonlike $\left(U^{82}\right)$ and heliumlike $\left(U^{90+}\right)$ ionization states, the $2 s_{1 / 2} \rightarrow 2 p_{3 / 2}$ transitions have energies near $4.5 \mathrm{keV}$, suitable for high-resolution study with a crystal spectrometer.

We measured the energies of 13 of these transitions in uranium ions trapped and ionized in Super-EBIT, with the electron beam energy set between 100 and $125 \mathrm{keV} .^{17}$ The $\mathrm{x}$-rays were analyzed by a von Hámos geometry crystal spectrometer. A spectrum taken under "high" charge-state conditions is shown in Figure 9a. The photon energies were calibrated using lines from hydrogenic and heliumlike $\mathrm{K}^{18+}$ and $\mathrm{K}^{17+}$ (shown in Figure 9b), hydrogenic $\mathrm{Cu}^{28+}$, and neonlike $\mathrm{Xe}^{4+4}$. Data were taken by alternating between uranium and calibration spectra to guard against any instrumental drifts.

The measured energy of the lithiumlike transition, $4459.37 \pm 0.35 \mathrm{eV}$, is in excellent
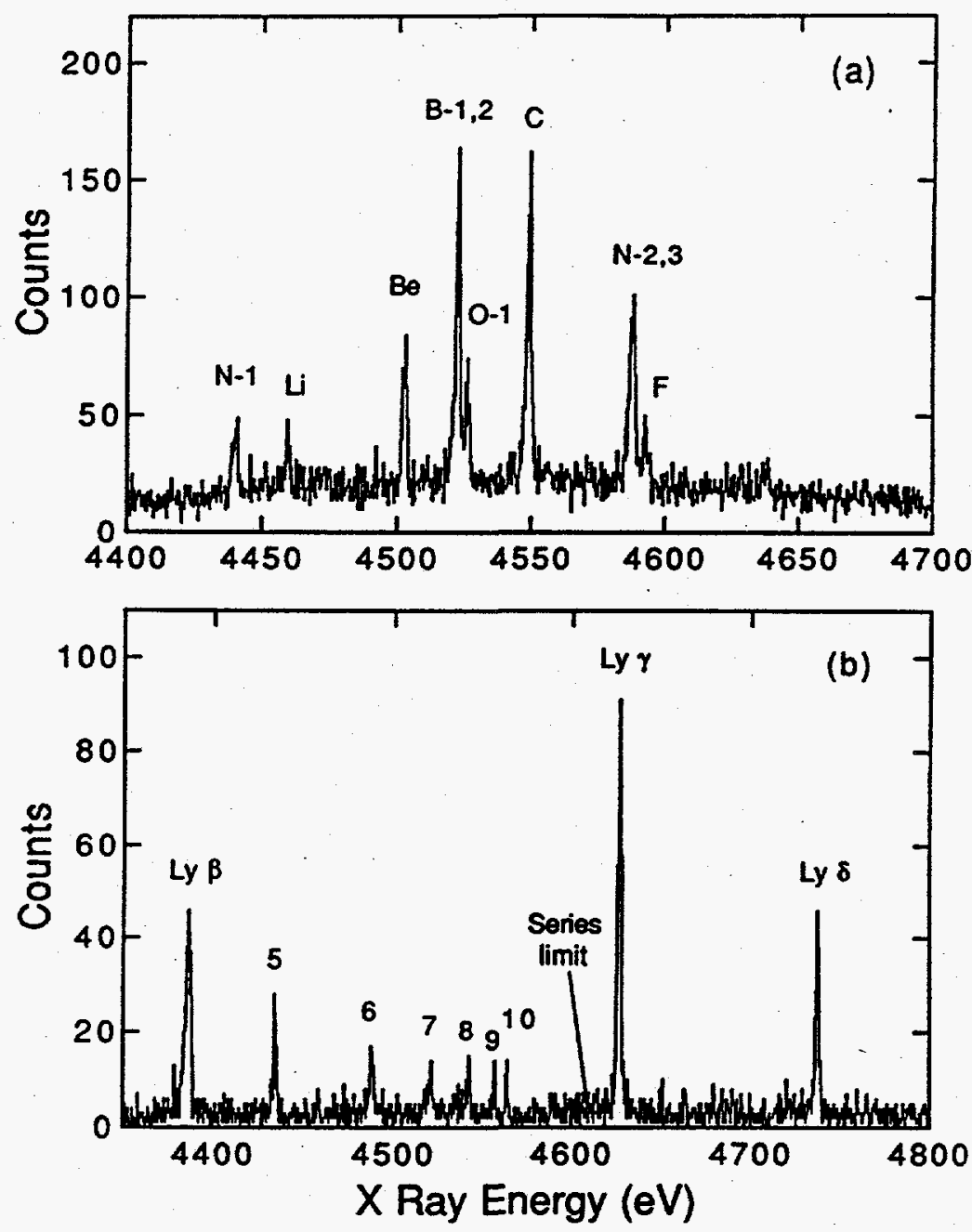

Figure 9. (a) Crystal spectrometer spectrum of highly-charged $U$ ions. The lines are identified by the ionization state that produced them. (b) Calibration spectrum of hydrogenic and heliumlike $K$ ions. 
agreement with theoretical predictions. The energies of the transitions in the other ionization states showed a systematic deviation from standard multiconfiguration Dirac-Fock calculations. ${ }^{18}$ Most of the differences between experiment and theory are attributable to electron-electron correlation effects. These results have prompted a new effort to calculate these transition energies via multibody perturbation theory. ${ }^{19}$

\section{Direct Binding Energy Measurements}

Precise measurements of the binding energies of states in few-electron high- $Z$ ions can provide stringent checks of QED and relativistic theory. In EBIT, such measurements are possible by use of photons emitted from radiative recombination. In this process, illustrated in Figure 10a, a beam electron is captured into a vacant state in the ion, emitting a photon with $E_{\gamma}$ given by

$$
E_{\gamma}=E_{\mathrm{e}}+E_{\mathrm{B}}
$$

where $E_{\mathrm{o}}$ is the electron-ion interaction energy and $E_{\mathrm{B}}$ is the binding energy of the state to which the electron recombines. The EBIT technique consists of observing the radiative recombination lines from the ions of interest using a solid-state detector while simultaneously observing radiative recombination from bare and hydrogenic low- $Z$ ions and gamma rays of known energies from radioactive sources. ${ }^{20}$ The gamma rays provide an accurate energy calibration for the detector, and the low- $Z$ radiative recombination lines supply an independent measurement of the electron-ion interaction energy. Because all the photons are recorded simultaneously, systematic errors resulting from electronic shifts or electron beam

(a)

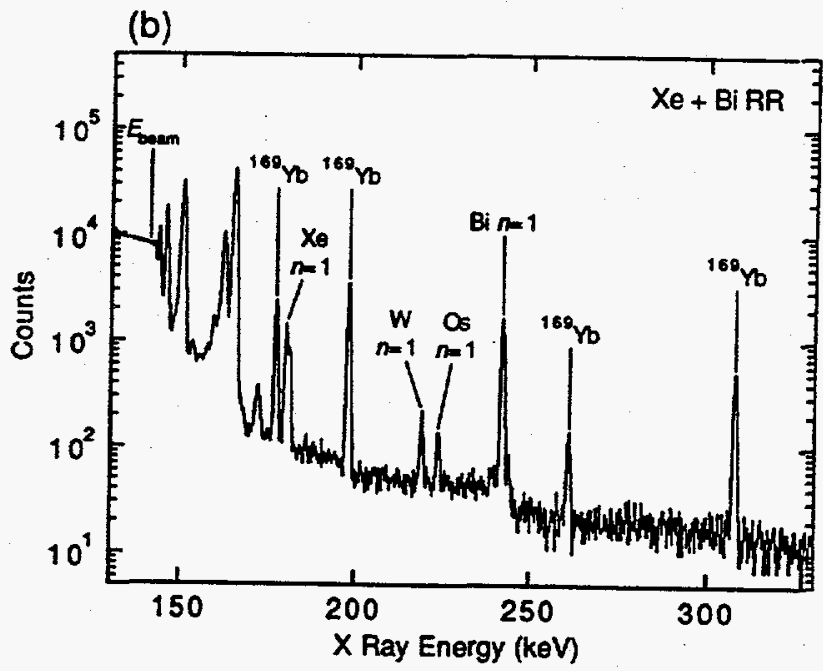

Figure 10 (a) Schematic representation of radiative recombination. A beam electron is captured into a vacant bound state of the ion, emitting a photon whose energy is equal to the sum of the initial electron energy plus the binding energy of the state to which is recombines. (b) Binding-energy data for Xe and Bi $n=1$ states, calibrated by a ${ }^{169} \mathrm{Yb}$ gamma-ray source. 
energy variations are eliminated.

The ions in EBIT are in their ground state configurations, so the interpretation of the binding energies measured using this technique is quite simple. One spectrum is shown in Figure $10 \mathrm{~b}$. An ultimate accuracy of about $10 \mathrm{eV}(100 \mathrm{ppm})$ should be achievable by this method.

\section{ION-ELECTRON INTERACTION MEASUREMENTS}

Aside from spectroscopic measurements, the most prolific area of research at EBIT has been that of ion-electron interactions. The first experimental result from EBIT was a measurement of the electron impact excitation cross sections for $n=3-2$ transitions in neonlike $\mathrm{Ba}^{45+} \cdot{ }^{21}$ EBIT provides a good environment to study these interactions; the ions in the trap are constantly bombarded by a stream of nearly monoenergetic electrons. We have made use of a variety of techniques to extract information about the interaction cross sections. These experiments have frequently been complementary to those performed with other ion sources, a result of the different information gathered.

\section{Ionization Cross Sections}

Several techniques have been developed for the measurement of electron impact ionization cross sections at EBIT. ${ }^{22}$ I will describe two of the most successful. The first, developed by Keith Wong and collaborators, uses the high rate of dielectronic recombination to correlate ionizations with recombination events in lithiumlike ions. The measurement

(a)

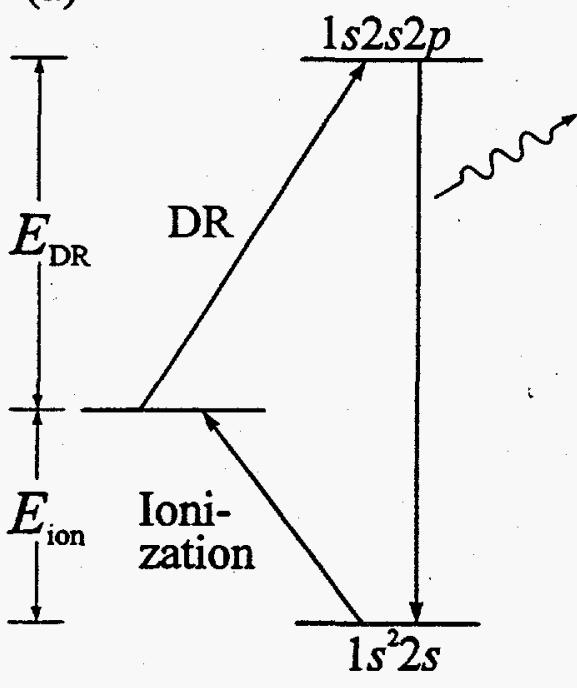

(b)

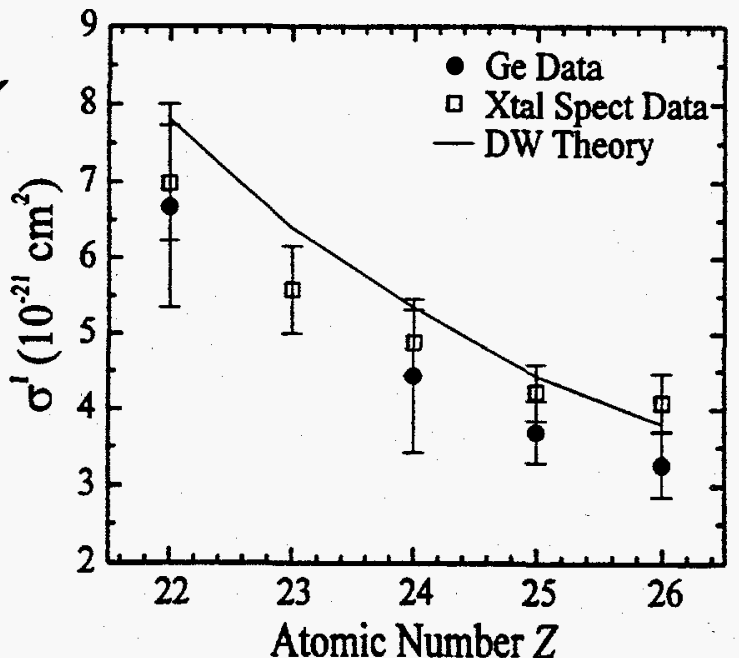

Figure 11. (a) Measurement scheme for ionization cross sections using dielectronic recombination photons. Ionization of a lithiumlike ion creates a heliumlike ion, which undergoes dielectronic recombination and emits a photon. (b) Results of these measurements, using a Ge detector (circles) and a crystal spectrometer (squares). The solid line is a distorted-wave theoretical prediction using the code described in Ref. 24. 
scheme is shown in Figure 11a. When the electron-ion interaction energy is at a dielectronic recombination resonance for heliumlike ions, the ionization of a lithiumlike ion results in $n=2-1 \mathrm{x}$-ray emission. At a steady-state equilibrium, the heliumlike ionization cross section $\sigma_{\mathrm{He}}^{\mathrm{I}}$ is given by

$$
\sigma_{\mathrm{He}}^{\mathrm{I}}=\frac{I_{\mathrm{DR}}}{I_{\mathrm{RR}}} \frac{\sigma_{\mathrm{Li}}^{\mathrm{RR}}}{A_{1}}\left[1+\frac{\sigma_{\mathrm{H}}^{\mathrm{RR}}}{\sigma_{\mathrm{He}}^{\mathrm{DR}}}+\frac{n_{0} v_{0} e}{\left\langle j_{e}\right\rangle} \frac{\sigma_{\mathrm{He}}^{\mathrm{CX}}}{\sigma_{\mathrm{He}}^{\mathrm{DR}}}\right] \times\left[1+\frac{\sigma_{\mathrm{He}}^{\mathrm{RR}} N_{\mathrm{He}}}{\sigma_{\mathrm{L}}^{\mathrm{RR}} N_{\mathrm{L}}}+\frac{\sigma_{\mathrm{B}}^{\mathrm{RR}} N_{\mathrm{Be}}}{\sigma_{\mathrm{Li}}^{\mathrm{RR}} N_{\mathrm{L}}}\right],
$$

where $I_{\mathrm{DR}}$ is the dielectronic recombination signal in a solid-state detector, $I_{\mathrm{RR}}$ is the total radiative recombination signal, and $A_{1}$ is the angular distribution factor for the dielectronic recombination photons. For these measurements, the ratios $N_{\mathrm{Hd}} / N_{\mathrm{Li}}$ and $N_{\mathrm{Bd}} / N_{\mathrm{Li}}$ were obtained from the appropriate ratios of the heliumlike line $w$, the lithiumlike line $q$, and the berylliumlike line $\beta$ observed with a crystal spectrometer. This technique essentially normalizes the cross section to the theoretically calculated radiative recombination cross section. The cross section can also be determined from the crystal spectrometer alone, in which the radiative recombination photons are not observed, by normalizing to theoretical cross section for excitation of the lithiumlike line $q$ :

$$
\sigma_{\mathrm{He}}^{1}=\frac{I_{\mathrm{DR}}}{I_{q}} \frac{\beta_{r} \sigma_{\mathrm{L}}^{q} W_{q}}{W_{\mathrm{DR}}}\left[1+\frac{\sigma_{\mathrm{He}}^{\mathrm{RR}}}{\sigma_{\mathrm{He}}^{\mathrm{DR}}}+\frac{n_{0} \nu_{0} \mathrm{e}}{\left\langle j_{e}\right\rangle} \frac{\sigma_{\mathrm{He}}^{\mathrm{CX}}}{\sigma_{\mathrm{Hk}}^{\mathrm{DR}}}\right]
$$

Here $I_{\mathrm{DR}}$ and $I_{q}$ are the detected intensities of the dielectronic recombination satellites and line $q$, and $\beta_{r}$ is the radiative branching ratio of the excited state that leads to line $q$.

This technique has been to measure the ionization cross sections for lithiumlike ions with $22 \leq \mathrm{Z} \leq 26^{23}$. The results are shown in Figure 11b, and compared to theoretical predictions. ${ }^{24}$ This approach has also been used to measure ionization cross sections in lithiumlike $\mathrm{Ba}^{33+}$, with somewhat worse agreement with theory, but larger uncertainties. ${ }^{25}$

Another technique, developed by Ross Marrs, is optimal for hydrogenic and heliumlike high- $Z$ ions, for which the $n=1$ radiative recombination photons are resolvable by a solid-state detector. From the observed intensities of the radiative recombination photons, the ratio $N_{\text {bard }} / N_{\mathrm{H}}$ is obtained, and the ionization cross section for the hydrogenic ions, $\sigma_{\mathrm{H}}^{\mathrm{g}}$, can be determined by

$$
\sigma_{\mathrm{H}}^{1}=\frac{N_{\text {bare }}}{N_{\mathrm{H}}}\left(\sigma_{\text {bare }}^{\mathrm{RR}}+\left\langle\sigma_{\text {barc }}^{\mathrm{CX}}\right\rangle\right),
$$

where $\left\langle\sigma_{b a r c}^{\alpha}\right\rangle=\left(\mathrm{e} /\left\langle j_{e}\right\rangle\right) n_{0} v_{0} \sigma_{b a r e}^{\alpha}$ is an effective charge-exchange recombination cross section. Measurements at different values of $n_{0}$ can provide an extrapolation of the charge-exchange to $n_{0}=0$ if required. A similar calculation provides the ionization cross section for heliumlike ions using, additionally, the ratio $N_{\mathrm{H}} / N_{\mathrm{He}}$.

This method has been used to measure the ionization cross sections for heliumlike $\mathrm{U}^{90+}$ and hydrogenic $\mathrm{U}^{91+26}$ The recorded photon spectrum, showing the radiative recombination photons from several ionization states, including bare $\mathrm{U}^{92+}$, is shown in Figure 12. The resulting cross sections, $\sigma_{\mathrm{H}}^{\mathrm{H}}=1.55 \pm 0.27 \mathrm{~b}$ and $\sigma_{\mathrm{He}}^{\mathrm{I}}=2.82 \pm 0.35 \mathrm{~b}$, are in reasonably good agreement with theoretical calculations ${ }^{24}$ but disagree significantly with previous measurements. ${ }^{27}$ 


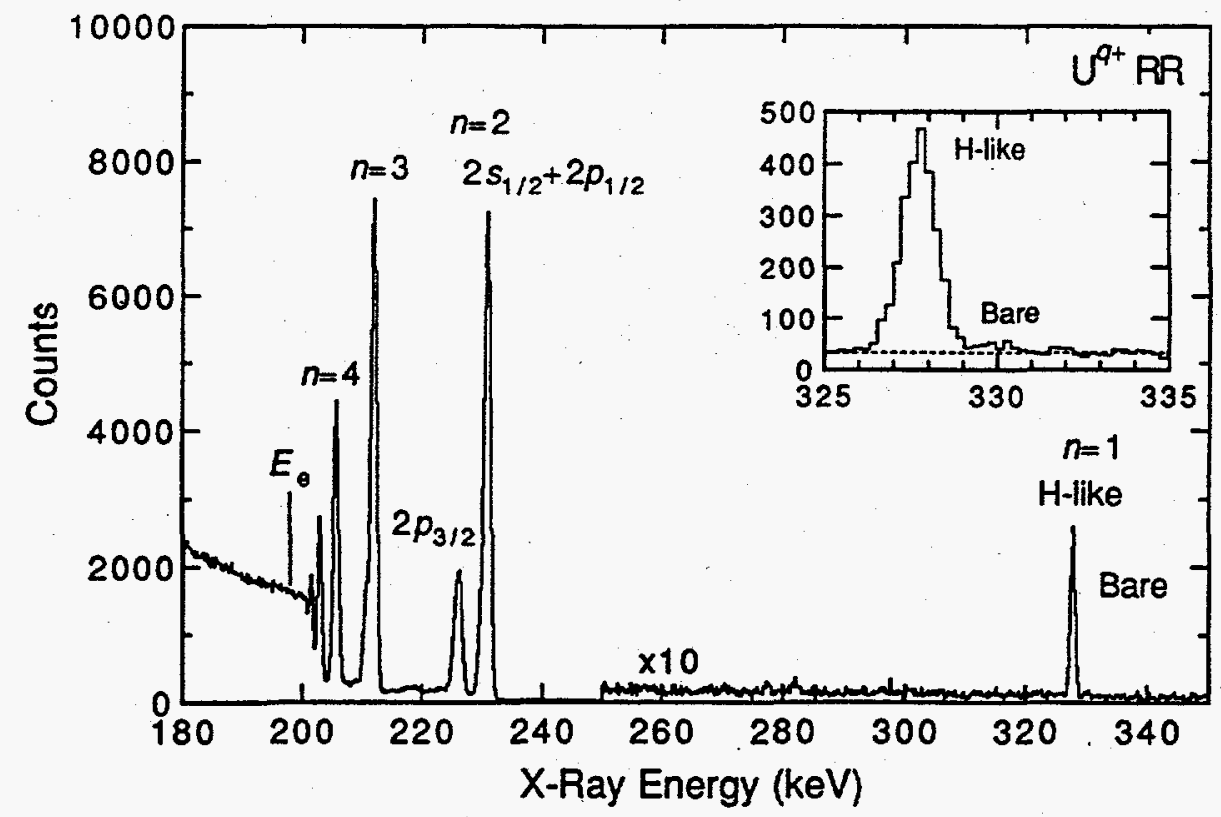

Figure 12. Spectrum of uranium ions in Super-EBIT taken at a beam energy of $198 \mathrm{keV}$. The $n=1$ radiative recombination features from the bare and hydrogenic ionization states are expanded in the inset.

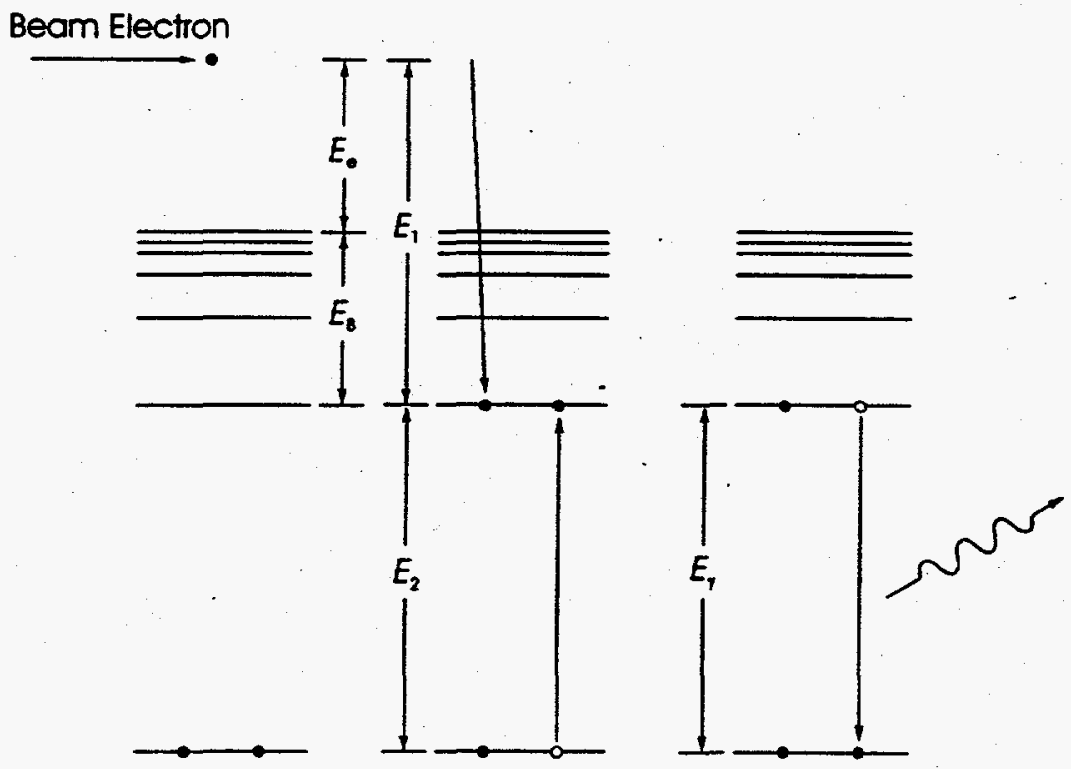

Figure 13. Schematic view of the process of dielectronic recombination. A beam electron is captured into a vacant state of an ion, exciting an electron already present in the ion. The resulting intermediate doubly-excited state decays radiatively. 


\section{Dielectronic Recombination Cross Sections}

Dielectronic recombination is an important recombination mode in plasmas. The process of dielectronic recombination, illustrated in Figure 13, has two steps. First, an electron is captured from the continuum into an excited state of the ion, much as in radiative

(a)
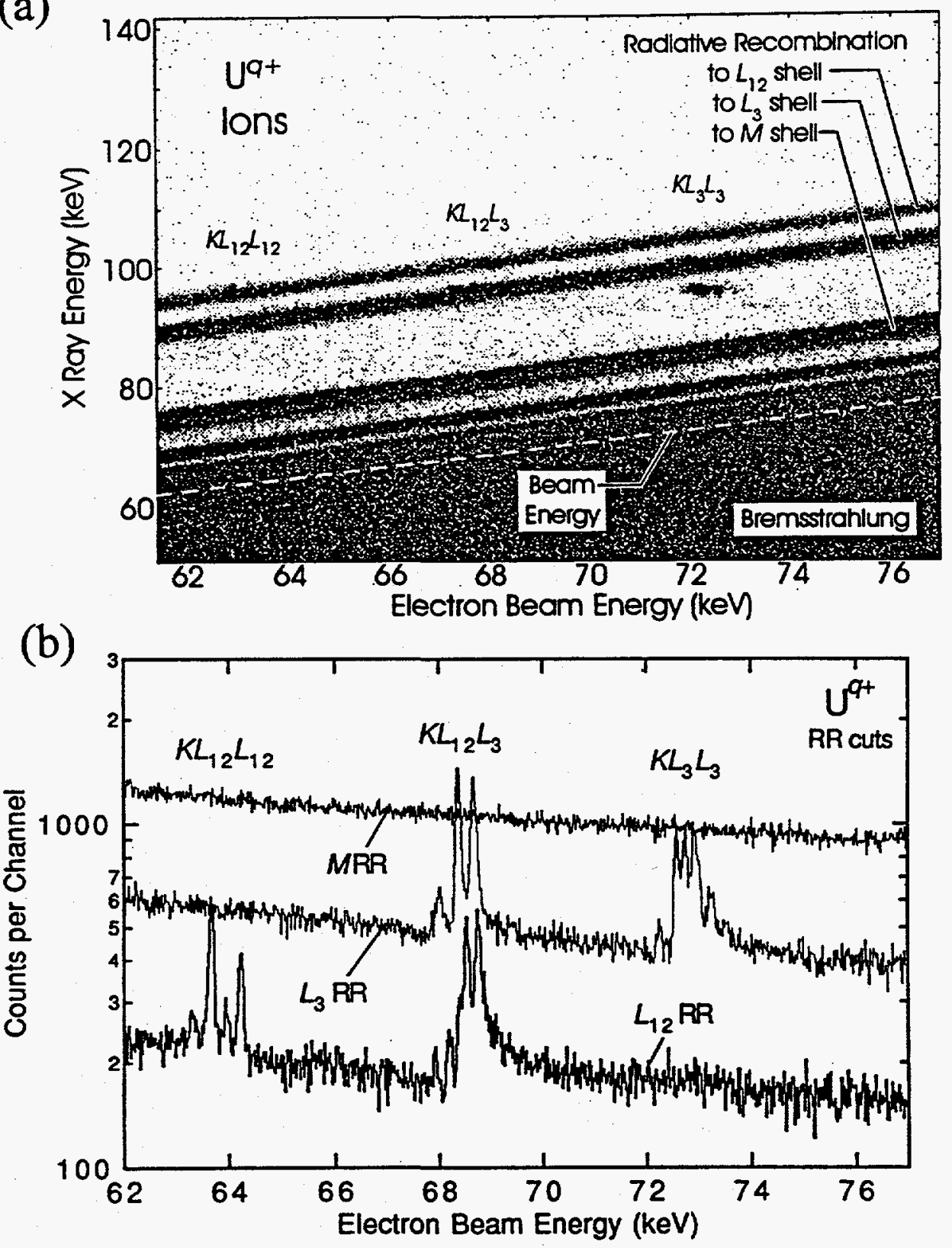

Figure 14. (a) Scatter plot of dielectronic recombination data for near-heliumlike uranium ions. Radiative recombination photons form angled bands, while dielectronic recombination photons appear as intense spots. (b) Projection of data from the $M, L_{3}$, and $L_{12}$ radiative recombination bands onto the electron beam energy axis. The dielectronic recombination resonances are apparent on top of the $L$-shell recombination. 
recombination. However, rather than emission of a photon, the process excites another electron, already present in the ion, from one bound state to another in an inverse Auger process. The process is resonant for

$$
E_{\mathrm{e}}+E_{\mathrm{B}}=E_{12}
$$

where $E_{B}$ is the binding energy of the state to which the beam electron is captured and $E_{12}$ is the energy difference between the initial and final states of the bound electron. The resulting doubly-excited intermediate state may decay either by Auger emission or by radiation; if the latter occurs, the entire process is dielectronic recombination. The dielectronic recombination resonances are labeled in a manner analogous to Auger lines; the $K L L$ resonances are those for which an electron is captured into the $L$ shell while a bound electron is excited from the $K$ shell to the $L$ shell.

Dielectronic recombination has been measured at EBIT with a number of experimental approaches. ${ }^{28,29}$ I will describe some of the most recent results, in which the capabilities of EBIT are used to the best advantage.

The primary technique for observing dielectronic recombination at EBIT has been the observation of the emitted photons. In order to prevent the recombination from substantially altering the ionization balance in the trap, the electron-ion interaction energy must be swept quickly over the resonances. In a typical dielectronic recombination experiment, ions are injected into the trap and ionized to the desired charge state. The electron-ion energy is then ramped linearly over the dielectronic recombination resonances, rapidly enough to avoid significant recombination. During the sweep, the photon energy, electron beam energy, and time are recorded for each detected photon.

A scatter plot of such data taken in the vicinity of the $K L L$ resonances for nearheliumlike uranium ions is shown in Figure 14a. The radiative recombination, dielectronic recombination, and bremsstrahlung features are labeled. Cuts may be applied to these data to separate the final states of the emitting transitions. Projections of the data onto the electron beam energy axis for cuts around the $M, L_{3}\left(2 p_{3 / 2}\right)$ and $L_{12}\left(2 s_{1 / 2}\right.$ and $\left.2 p_{1 / 2}\right)$ bands are shown in Figure 14b. A measurement of the total recombination cross section would record the sum of these three bands plus recombination to higher $n$ levels; the EBIT technique improves the signal-to-background ratio.

The recombination resonances in the $K L_{12} L_{3}$ manifold in the $L_{12}$ cut are visibly asymmetric. These data represent the first observation of quantum interference between radiative recombination and dielectronic recombination. ${ }^{30}$ The two processes have the same initial and final states (see Figure 13 and Figure 14), so interference is possible. The shape of the resonance peaks in the presence of significant interference should be a Fano profile: ${ }^{11}$

$$
\sigma^{\mathrm{DR}+\mathrm{RR}}(E)=\sigma_{a} \frac{(q+\varepsilon)^{2}}{1+\varepsilon^{2}}+\sigma_{b},
$$

where $\varepsilon=\left(E-E_{0}\right) /(\Gamma / 2), E_{0}$ is the resonance energy, $\Gamma$ is the Lorentzian width of the resonance, $\sigma_{a}$ and $\sigma_{b}$ are the interfering and non-interfering parts of the continuum cross section, and $q$ is the Fano lineshape factor. A comparison of fits to the data using Lorentzian and Fano lineshapes is shown in Figure 15. The $q$ values derived from this fit are in good agreement with the minimum $q$ values possible from the resonance strengths of the largest resonances for each ionization state, indicating maximal interference. 
Our data provide additional evidence that the observed asymmetry is the result of interference rather than an instrumental effect. We compared the resonance shape observed for the sweep from high to low beam energy with that observed for the sweep from low to high beam energy; no difference was observed. The smoothness of the projection of the $M$ radiative recombination photons (see Figure 14b) indicates that the ion-beam overlap did

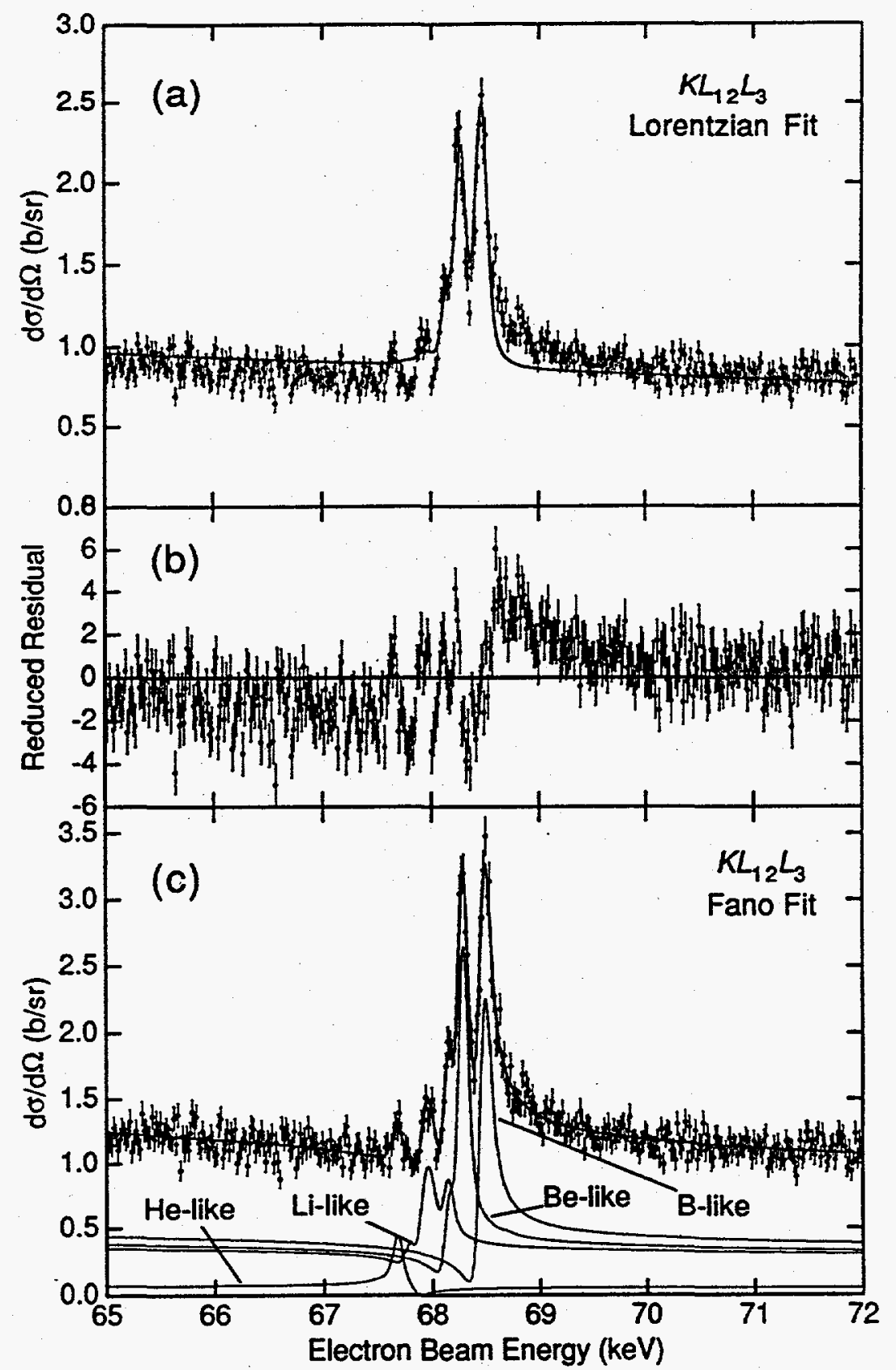

Figure 15. (a) Fit of the $K L_{12} L_{3}$ resonance data using a Lorentzian lineshape. (b) Reduced residuals from Lorentzian fit. The systematic variation is quite obvious. (c) Fit of the same data using a Fano profile. The $\chi_{\nu}^{2}$ values for the two fits are 2.98 and 1.02 , respectively. For these fits, the resonance widths, relative positions, and relative intensities were fixed to theoretical predictions, and one value of the Fano parameter $q$ was fit for each ionization state. 
not change over the resonance interval. We have also measured these resonances in nearheliumlike bismuth ions, and see a similar signature of interference.

\section{ION-SURFACE INTERACTION STUDIES}

After the initial successes of experiments observing ions in situ in EBIT, Dieter Schneider and his collaborators built an ion-extraction system for EBIT to allow experiments with ions outside the trap. ${ }^{7}$ The ions can be extracted from the trap with energies as low as $2 \mathrm{keV} \times q$ and charge-state analyzed by a dipole bending magnet. This system allows investigation of the region in which the potential energy of an ion is comparable to its kinetic energy. The interactions of these ions with surfaces have been the focus of much of the experimental work with the extracted ions.32

\section{Electron Emission from Surfaces}

A highly-charged ion incident on a surface can produce electrons via kinetic emission, originating in the kinetic energy of the ion, and potential emission originating in the potential energy of the ion. These processes are the subject of much experimental and theoretical interest. As a slow highly-charged ion approaches a conducting surface, the potential barrier for transfer of electrons from the material to the ion drops below the Fermi level at a distance $r_{c}$ given by:

$$
r_{c}=\frac{1}{2 W_{\Phi}} \sqrt{8 q+2} \approx \frac{\sqrt{2 q}}{W_{\Phi}}(\text { for } q>>1)
$$

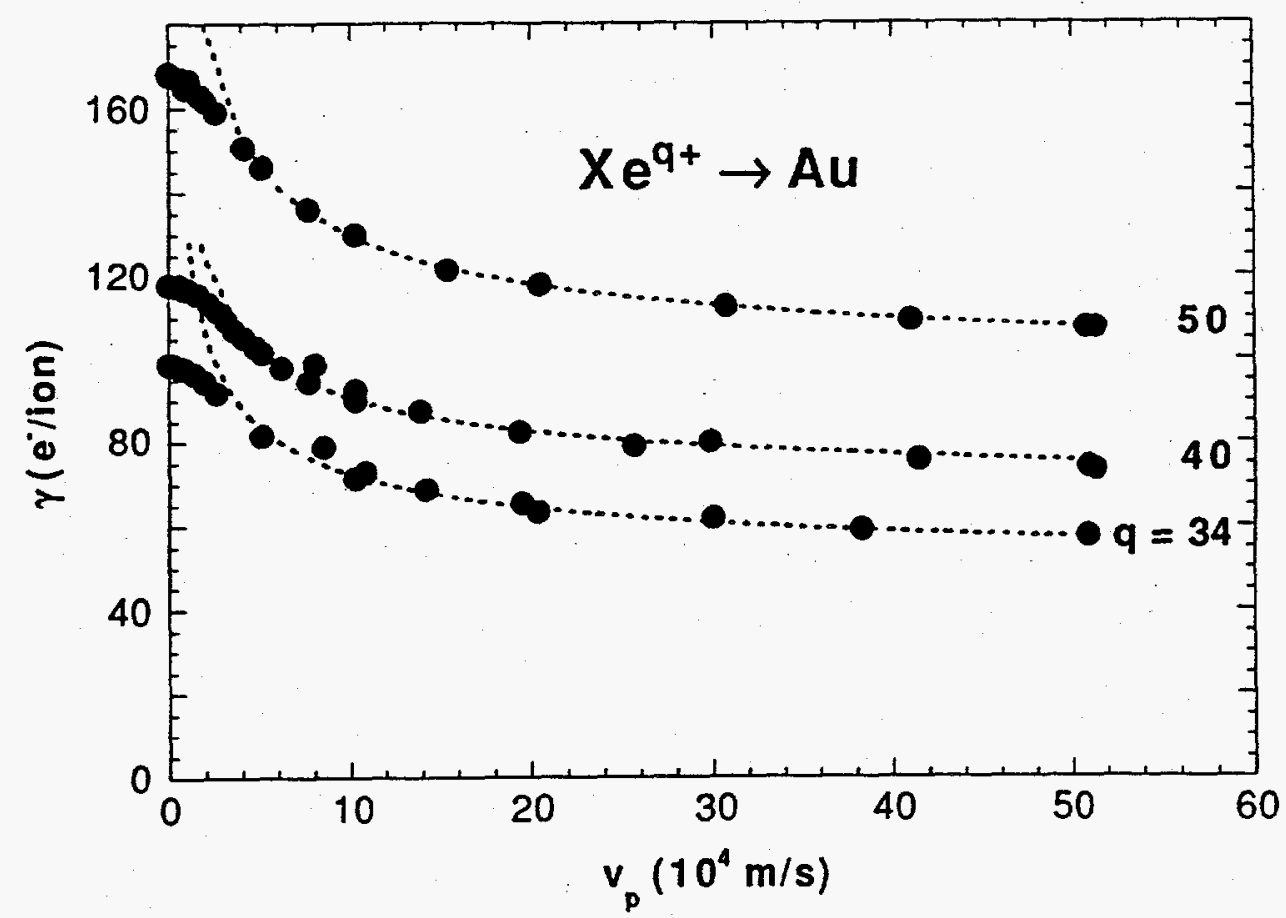

Figure 16. Number of electrons emitted per $\mathrm{Xe}^{\mathrm{st}}$ ion incident on a gold surface as a function of nominal ion velocity. The dashed lines are fits to points with $v_{p}>2.5 \times 10^{6} \mathrm{~cm} / \mathrm{s}$ using Equation (10). The deviation from the fit at low $v_{p}$ is a result of image charge acceleration of the ions. 
where $q$ is the ion charge and $W_{\Phi}$ is the work function of the surface material..$^{33}$ At this point, electrons are resonantly captured into high-n states of the ion, forming a "hollow atom." This object gives rise to electron emission by a variety of processes, including autoionization, Auger loss to the conduction band, resonant ionization to the conduction band, and secondary electron emission from higher-energy Auger electrons and soft $\mathrm{x}$ rays emitted as the ion enters the surface. Early studies using EBIT ions indicated that the number of emitted electrons per ion is quite high for highly charged heavy ions, though not as high as would be expected by extrapolation from low- $Z$ results. ${ }^{34}$

Fritz Aumayr and his colleagues have recently performed a systematic study of electron yields from slow $\mathrm{Ar}^{q^{+}}, \mathrm{Xe}^{q+}$, and $\mathrm{Th}^{q+}$ ions normally incident on a clean gold surface. ${ }^{35}$ The ions were produced in EBIT, extracted and decelerated to velocities $\nu_{\mathrm{p}}<5 \times 10^{7} \mathrm{~cm} / \mathrm{s}$. The electrons emitted from the surface were accelerated and detected in a solid-state surface-barrier detector.

A plot of the number of electrons emitted $(\gamma)$ as a function of the velocity of the incoming ion is shown in Figure 16. For ions with velocity $\nu_{p}>2.5 \times 10^{6} \mathrm{~cm} / \mathrm{s}$, the data are well represented by an empirical formula derived from earlier studies: ${ }^{36}$

$$
\gamma=\frac{c_{1}}{\sqrt{v_{p}}}+\gamma_{\infty}
$$

where $c_{1}$ is an empirical constant and $\gamma_{\infty}$ is the velocity-independent emission. At very low impact velocities, however, the data deviate significantly from the fit, a result of acceleration of the ions by the induced image charge in the surface. In fact, this deviation can be used to estimate the image charge acceleration, which was found to be in good agreement with theoretical predictions. ${ }^{33}$ The image-charge acceleration can, in turn, be used to estimate the value of $r_{c}$, the distance from the surface at which the first resonant electron transfer occurs. These derived values also agree well with theoretical predictions.

\section{Ion-Induced Surface Defects}

In a conductor, electrons move freely, so the charges required to neutralize an incident ion are readily available. The situation is quite different in the case of an insulator; the charges to neutralize an ion must all come from the atoms near the point of the ion's impact. Low-energy, highly charged ions do not significantly penetrate solid material, so the interaction of such an ion with an insulating surface will result in the deposit of a large amount of charge and energy in a small volume and would be expected to result in observable changes to the surface.

Dieter Schneider and his colleagues have observed such features from the impact of slow highly charged ions extracted from EBIT onto insulating surfaces. ${ }^{37}$ Cleaved mica $\left(\mathrm{KAl}_{3} \mathrm{Si}_{3} \mathrm{O}_{10}(\mathrm{OH})_{2}\right)$, Lexan $\left(\mathrm{C}_{16} \mathrm{H}_{14} \mathrm{O}_{3}\right)$, and silica glass $\left(\mathrm{SiO}_{2}\right)$ were exposed to a variety of ions, ranging from $\mathrm{Kr}^{35+}$ to $\mathrm{Th}^{24+}$, with energies of $7 \times q \mathrm{keV}$. After irradiation, the surfaces were imaged with an atomic force microscope with a resolution of about $1 \mathrm{~nm}$. The surfaces showed characteristic mounds 0.3 to $2 \mathrm{~nm}$ high and 5 to $100 \mathrm{~nm}$ in diameter. Repeated scans with the atomic force microscope occasionally "peeled" off the top layer, revealing a pit beneath the defect; thus, the structure is that of a "blister."

One image of several such blisters created by $490-\mathrm{keV} \mathrm{U}^{70+}$ ions on mica is shown in Figure 17 . The observed volume appears to be roughly linearly related to the ion charge. The detailed mechanisms for defect generation are still poorly understood, and are likely to be the subject of significant study in the future. 


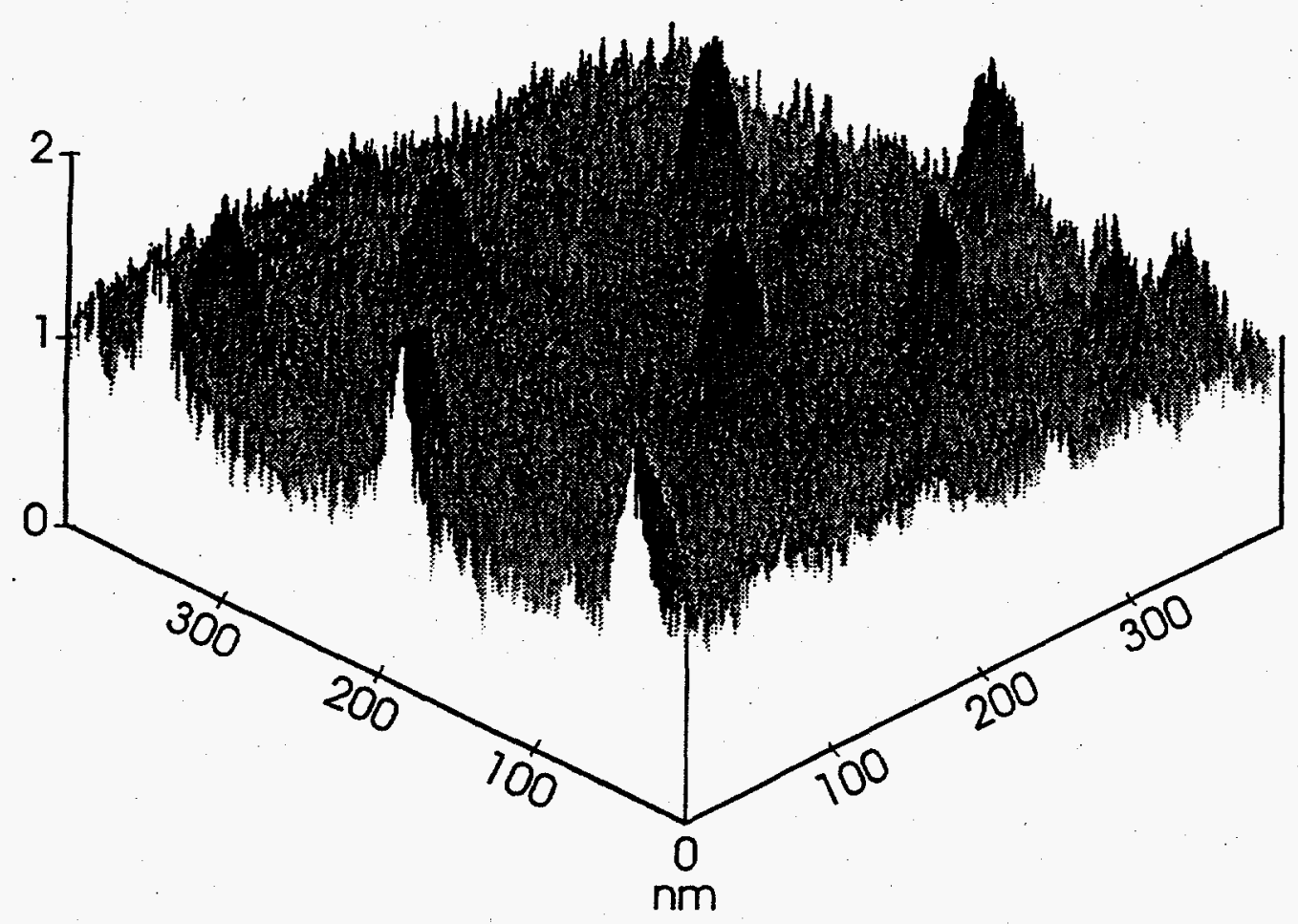

Figure 17. Atomic force microscope image of "blisters" created by the impact of $490-\mathrm{keV} \mathrm{U}^{\text {po+ }}$ ions on mica.

\section{OTHER EXPERIMENTS USING EBITS}

The EBIT traps are "active" traps; that is, the ions in the trap are constantly being bombarded by the beam electrons. While this property has some advantages, it does not allow the observation of the ions in the absence of the electrons. Two techniques that circumvent this problem have recently been developed.

\section{FT-ICR Observations}

If the electron beam is turned off, the ions in the EBIT trap follow circular cyclotron orbits with radii much larger than the electron beam diameter. Because the magnetic field in the trap volume is very uniform, it is possible to detect the ions by exciting them at their characteristic cyclotron frequencies and measuring the response. Peter Beiersdorfer and his coworkers have successfully detected this signal by means of Fourier-transform ion cyclotron resonance (FT-ICR) spectrometry. ${ }^{38}$

Two radio-frequency probes were inserted into the Super-EBIT via radial slots normally used as $x$-ray windows. One probe was used to excite the ions, while the other was used to detect the signal. After the electron beam was turned off, the ions were excited by a 25- $\mu$ s sine wave with a frequency near the cyclotron resonance of the ion to be observed. After a short time interval (a few ms) the detection probe measured the signal from the ions, which was Fourier-transformed into the frequency domain. Observed FT-ICR signals from krypton-84 ions from heliumlike $\mathrm{Kr}^{34+}$ to bare $\mathrm{Kr}^{36+}$ are shown in Figure 18. 


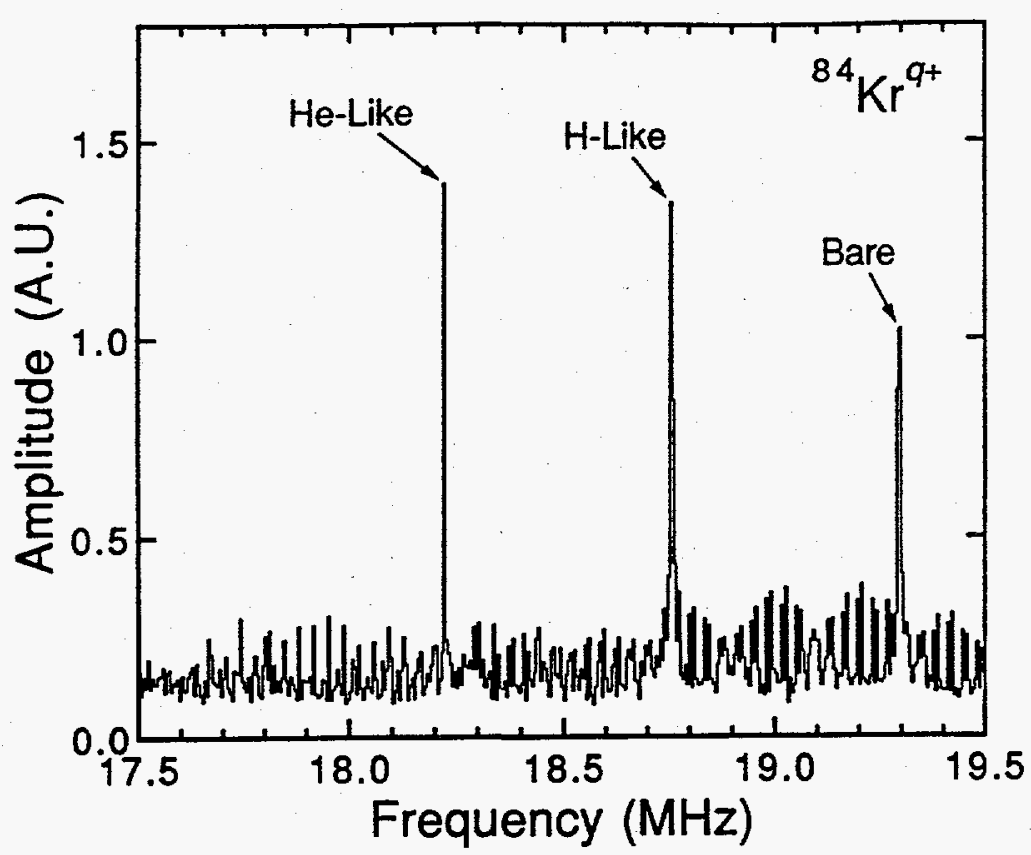

Figure 18. Fourier-transform ion cyclotron resonance spectrum of ${ }^{84} \mathrm{Kr}$ ions in Super-EBIT. The resolution is limited by the sampling time.

The FT-ICR technique has many possible applications. Most significant is the possibility of detecting the number of ions in the trap, to allow absolute cross section measurements. Even with the relatively crude probe arrangement used in the first experiments, as few as 1000 ions could be detected; with improvements, including the installation of a segmented drift tube optimized to detect the ICR signal, the sensitivity could be increased to detect as few as.10 ions, making experiments searching for very small signals possible.

\section{RETRAP}

Another option for observation of ions without the interference of the electron beam in EBIT is to transport the ions from EBIT into a separate trap designed for ions only. Just such a trap has been built by Dieter Schneider, David Church, and their group at LLNL. ${ }^{39}$ Dubbed RETRAP, the device is a cryogenic Penning trap with superconducting magnetic coils capable of operation to $6 \mathrm{~T}$. A schematic diagram of EBIT, the ion transport system, and RETRAP is shown in Figure 19. Ions are extracted from EBIT and transported to RETRAP via the ion-transport system, which includes a $90^{\circ}$ analyzing magnet to select a single charge state and several steerers and lenses. The Penning trap itself consists of five electrodes: two endcaps, a segmented ring electrode, and two compensation electrodes. The axial frequency of an ion in the trap, $\omega_{2}$, is given by:

$$
\omega_{z}^{2}=C_{2} \frac{2 q \mathrm{eV}}{M\left(z_{0}^{2}+r_{0}^{2} / 2\right)},
$$

where $z_{0}$ and $r_{0}$ are the half-length and radius of the trap, $V_{0}$ is the voltage applied to the endcap electrodes, $M$ and $q$ are the mass and charge of the ion in question, and $C_{2}=0.5449$. A high- $Q$ tuned circuit is attached to the compensation electrodes. If the axial frequency of 
the ions is set to the frequency of the tuned circuit, the ions can be detected by the induced noise. The circuit simultaneously cools the ions via radiation.

Recently, highly charged ions, including $\mathrm{Xe}^{4+}$ and $\mathrm{Th}^{72+}$, have been successfully introduced into RETRAP from EBIT. The ions were detected in the trap by a variety of methods, and have been extracted from RETRAP after being held for several seconds. Radiative cooling of the ions has been observed. The lifetime of uncooled $\mathrm{Xe}^{4+}$ ions in the trap is greater than $25 \mathrm{~s}$, in agreement with predictions.

Many exciting experiments are made possible by the RETRAP system. We plan to measure ion-atom and ion-ion collisions at very low energies, perform precision laser spectroscopy and lifetime measurements, and observe Wigner crystallization of the onecomponent strongly-coupled plasma formed by the ions.

\section{CONCLUSION}

The electron beam ion trap is a remarkably useful tool for performing diverse measurements with highly charged ions. We have built two such devices at the Lawrence Livermore National Laboratory; EBIT and Super-EBIT. With these, we have created and trapped ions up to bare $\mathrm{U}^{92^{+}}$, and made a wide variety of measurements on diverse systems. We continue to develop the devices to extend the range of possible experiments with them.

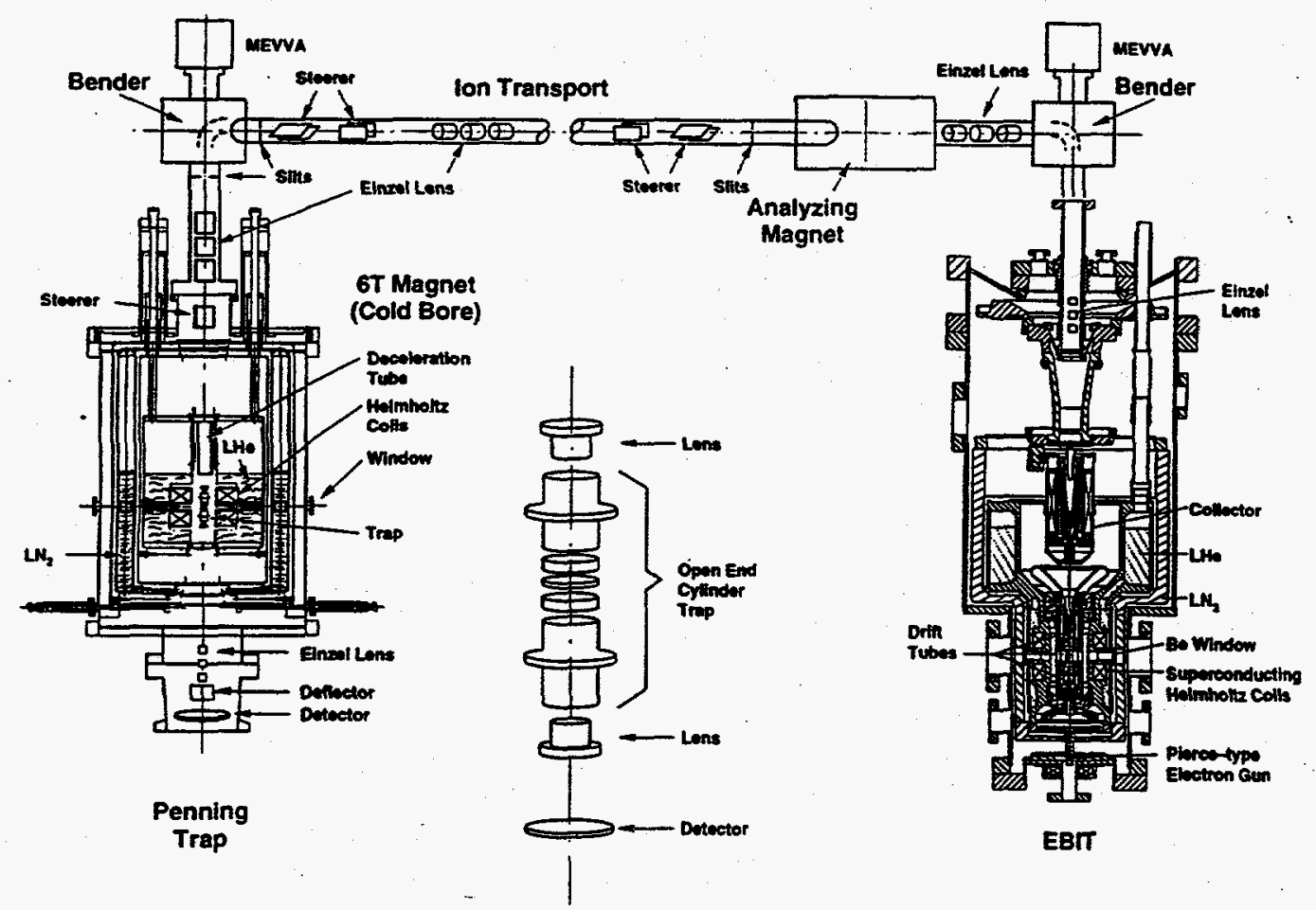

Figure 19. Layout of EBIT, transport system, and RETRAP. Ions created in EBIT are injected into RETRAP, where they can be studied without interference from the electron beam. 


\section{ACKNOWLEDGEMENTS}

This work was performed under the auspices of the U.S. Department of Energy by Lawrence Livermore National Laboratory under contract W-7405-ENG-48 and was funded in part by the DOE Office of Basic Energy Sciences under contract no. KB0403.

\section{REFERENCES}

1. M. A. Levine et al., Physica Scripta T22, 157 (1988); R: E. Marrs et al., Phys. Rev. Lett. 60, 1715 (1988); M. A. Levine et al., Nucl. Instr. Meth. B43, 431 (1989).

2. D. A. Knapp et al., Nucl. Instr. Meth. A334, 305 (1993).

3. S. R. Elliott and R. E. Marrs, submitted to Nucl. Instrum. Meth. (1994).

4. W. Lotz, Z. Phys. 216, 241 (1968).

5. Y:S. Kim and R.H. Pratt, Phys Rev A 27, 2913 (1983).

6. M. A. Levine et al., in International Symposium on Electron Beam Ion Sources and Their Applications, ed. A. Hershcovitch, AIP Conf. Proc. No. 188, (AIP, New York), p. 82 (1988); B. M. Penetrante et al., Phys. Rev. A 43, 4873 (1991).

7. D. Schneider et al., Phys. Rev. A 42, 3889 (1990); D. Schneider et al., Phys. Rev. A 44, 3119 (1991).

8. P. Beiersdorfer et al., Rev. Sci. Inst. 61, 2338 (1990).

9. P. Beiersdorfer et al., Phys. Rev. Lett. 67, 2272 (1991).

10. D. L. Matthews et al., Phys. Rev. Lett. 54, 110 (1985); T. Boehly et al., Phys. Rev. A 42, 6962 (1990); D. J. Fields et al., Phys. Rev. A 46, 1606 (1992).

11. A. V. Vinogradov, I. I. Sobelman, and E. A. Yukov, Sov. J. Quantum. Electron. 59, 5 (1975); B. A. Norton and H. J. Peacock, J. Phys. B 8, 989 (1975).

12. J. Nilsen et al., in $X$-Ray Lasers 1992, ed. E. E. Fill, IOP Conf. Proc. no. 125 (Institute for Physics, Bristol), p. 137 (1992).

13. P. Beiersdorfer, S. R. Elliott, and J. Nilsen, Phys. Rev. A 49, 3123 (1994).

14. B. J. Wargelin, P. Beiersdorfer, and S. M. Kahn, Phys. Rev. Lett. 71, 2196 (1993).

15. G. W. F. Drake, Phys. Rev. A 3, 908 (1971).

16. W. R. Johnson and C. Lin, Phys. Rev. A 9, 1486 (1974).

17. P. Beiersdorfer et al., Phys. Rev. Lett. 71, 3939 (1993).

18. I. P. Grant, et al., Comput. Phys. Commun. 21, 207 (1980).

19. W. R. Johnson et al., unpublished; W. R. Johnson, in these proceedings (1994).

20. R. E. Marrs, S. R. Elliott, and Th. Stohlker, in EBIT Annual Report 1993, University of California Report UCRL-ID-118274, p. 4 (1994).

21. R. E. Marrs et al., Phys. Rev. Lett. 60, 1715 (1988).

22. D. Vogel et al., Z. Phys. D 21, S193 (1991); P. Beiersdorfer et al.; Invited Papers, $X V I I$ ICPEAC, ed. W. R. MacGillivray, I. E. McCarthy, and M. C. Standage (Adam Hilger, NY) p. 313 (1992). 
23. K. L. Wong et al., Phys. Rev. A 48, 2850 (1993).

24. H. L. Zhang and D. H. Sampson, Phys. Rev. A 42, 5378 (1990); K. J. Reed (private communication).

25. K. L. Wong et al., Z. Phys. D 21, S197 (1991).

26. R. E. Marrs, S. R. Elliott, and D. A. Knapp, Phys. Rev. Lett. 72, 4082 (1994).

27. N. Claytor et al., Phys. Rev. Lett. 61, 2081 (1988).

28. D. A. Knapp et al., Phys Rev. A 47, 2039 (1993); D. A. Knapp, Recombination of Atomic lons, NATO ASI Series B 296, 181 (1992); M. B. Schneider et al., Phys. Rev. A 45, R1291 (1992).

29. D. R. DeWitt et al., Phys. Rev. A 44, 7185 (1991); D. R. DeWitt et al., Phys. Rev. Lett. 68, 1694 (1992).

30. D. A. Knapp et al., submitted to Phys. Rev. Lett. (1994).

31. U. Fano, Phys. Rev. 124, 1866 (1961); U. Fano and J. W. Cooper, Phys. Rev. 137, A1364 (1963).

32. D. Schneider et al., Radiation Effects and Defects in Solids, 127, 113 (1993).

33. J. Burgdörfer, P. Lerner, and F. W. Meyer, Phys. Rev. A 44, 5674 (1991); J. Burgdörfer and F. W. Meyer, Phys. Rev. A 47, R20 (1993).

34. J. W. McDonald et al., Phys. Rev. Lett. 68, 2297 (1992).

35. F, Aumayr et al., Phys. Rev. Lett. 71, 1943 (1993); H. Kurz et al., Phys. Rev. A 49, 4693 (1994).

36. H. Kurz et al., Phys. Rev. Lett. 69, 1140 (1992); H: Kurz et al., Phys. Rev. A 48, 2182 (1993).

37. D. Schneider et al., Surface Science 294, 403 (1993); D. Schneider et al., Nucl. Instrum. Meth. B 87, 156 (1994); M. Briere et al., Nucl. Instrum. Meth. B 90, 231 (1994); C. Rühlicke et al., submitted to Thirteenth International Conference on the Application of Accelerators in Research and Industry, Denton, TX (1994).

38. P. Beiersdorfer et al., Rapid Comm. in Mass Spect., 8, 141 (1994)

39. D. Schneider et al., Rev. Sci. Instrum. 65, (no. 11) (1994). 